TRANSACTIONS OF THE

AMERICAN MATHEMATICAL SOCIETY

Volume 350, Number 3, March 1998, Pages 1013-1044

S 0002-9947(98)01822-4

\title{
TIGHT CONTACT STRUCTURES ON SOLID TORI
}

\author{
SERGEI MAKAR-LIMANOV
}

\begin{abstract}
In this paper we study properties of tight contact structures on solid tori. In particular we discuss ways of distinguishing two solid tori with tight contact structures. We also give examples of unusual tight contact structures on solid tori.

We prove the existence of a $\mathbb{Z}$-valued and a $\mathbb{R} / 2 \pi \mathbb{Z}$-valued invariant of a closed solid torus. We call them the self-linking number and the rotation number respectively. We then extend these definitions to the case of an open solid torus. We show that these invariants exhibit certain monotonicity properties with respect to inclusion. We also prove a number of results which give sufficient conditions for two solid tori to be contactomorphic.

At the same time we discuss various ways of constructing a tight contact structure on a solid torus. We then produce examples of solid tori with tight contact structures and calculate self-linking and rotation numbers for these tori. These examples show that the invariants we defined do not give a complete classification of tight contact structure on open solid tori.

At the end, we construct a family of tight contact structure on a solid torus such that the induced contact structure on a finite-sheeted cover of that solid torus is no longer tight. This answers negatively a question asked by Eliashberg in 1990. We also give an example of tight contact structure on an open solid torus which cannot be contactly embedded into a sphere with the standard contact structure, another example of unexpected behavior.
\end{abstract}

\section{INTRODUCTION AND MAIN RESULTS}

1.1. Contact structures and orientations. A contact structure on a 3-manifold $M$ is a completely non-integrable tangent plane field $\xi$. A plane field is a kernel of some (locally defined) 1-form $\alpha$ such that $\xi=\{\alpha=0\}$. The choice of $\alpha$ is unique, up to a multiplication by a non-vanishing function $f$. The complete nonintegrability of $\xi$ is equivalent to the inequality $\alpha \wedge d \alpha \neq 0$. The form $\alpha \wedge d \alpha$ can be thought of as a choice of a volume element on $M$; this is equivalent to choosing an orientation of $\mathrm{T}(M)$. This orientation is independent of the choice of the one-form. In the case when $\alpha$ can be chosen globally, we can co-orient $\xi$ by choosing any vector field $R$, such that $\alpha(R)>0$. The co-orientation changes if we choose $-\alpha$ instead of $\alpha$. Thus a co-oriented contact structure corresponds to choosing a "ray" of 1 -forms $\mathcal{A}_{\xi}^{+}=\{f \cdot \alpha \mid f>0\}$. The choice of $\mathcal{A}_{\xi}^{+}$also allows us to orient the plane field $\xi$.

In this paper, we shall always assume that the contact structure is co-oriented and that $\xi$ and $M$ are oriented.

Received by the editors January 18, 1995.

1991 Mathematics Subject Classification. Primary 53C15; Secondary 57R15, 58F05.

(c)1998 American Mathematical Society 
1.2. Characteristic foliation of a surface and the germ of a contact structure near a surface. A generic surface $F^{2} \subset M^{3}$ is transversal to $\xi$ outside a set of isolated points, where it is tangent to $\xi$. Thus the intersection $\mathrm{T}(F) \cap \xi$ forms a line field which has isolated singularities at the points of tangency. This singular line field is called the characteristic foliation of the surface and is denoted by $F_{\xi}$. We shall also refer to the set of integral curves of this (singular) line field as the characteristic foliation as well. The points of tangency will be called the singularities of the characteristic foliation, and curves tangent to the foliation will be called the leaves of the characteristic foliation.

$F_{\xi}$ is always locally orientable. Therefore, the index of the line field is well defined at a singularity point. In the generic case, this index is equal to \pm 1 . A singular point will be called elliptic if the index is +1 and hyperbolic if the index is -1 . A leaf of the characteristic foliation which starts or terminates at a hyperbolic point is called a separatrix.

If $\mathrm{T}(F)$ is oriented or co-oriented, then it is possible to induce an orientation on the characteristic foliation. In this case the singularities of the characteristic foliation can be given signs by comparing the (co)orientation of $\xi$ with the (co)orientation of $\mathrm{T}(F)$. This convention means that positive elliptic points act as sources and negative elliptic points act as sinks (the sign of a hyperbolic point is harder to determine because it depends on $\mathcal{C}^{1}$ properties of the foliation) [El4]. We shall always assume that the characteristic foliation is oriented.

The (oriented) characteristic foliation $F_{\xi}$ uniquely defines the germ of a contact structure along $F$ (up to an isotopy which preserves $F_{\xi}$ ) [Gi1] (see also section 2).

1.3. Tight and overtwisted contact structures. A closed non-singular leaf of a characteristic foliation is called a cycle of the characteristic foliation. In 1982, Bennequin proved that for any disk $D \subset \mathbb{S}^{3}$ the characteristic foliation induced by the standard contact structure never contains a cycle $[\mathrm{Be}]$. He used this fact to distinguish the standard contact structure on the sphere from other, exotic contact structures. In general, a contact structure $\xi$ on a manifold $M$ will be called tight if for all $D \subset M, D_{\xi}$ has no cycles. The definition of tightness is obviously equivalent to the requirement that the characteristic foliation of any surface should have no cycles which are contractable in $F$. This places a severe restriction on the combinatorics of the characteristic foliation. We shall explore these restrictions in section 3. If there exists a disk $D \subset(M, \xi)$ such that $D_{\xi}$ does have a closed cycle then $\xi$ is called an overtwisted contact structure. The concepts of tightness and overtwistedness were introduced by Eliashberg in 1989 [El1], who gave a complete classification of overtwisted contact structures on closed manifolds. The nature of the relationship between overtwisted and tight contact structures is still not well understood, however. It is easy to see that a manifold with an overtwisted contact structure can never be covered by a manifold with tight contact structure. But it was not known if a manifold with tight contact structure can be covered by an overtwisted manifold. In this paper we construct a tight solid torus with an overtwisted cover, answering this question for the case of manifolds with boundary. As far as we know, this question is still open for the case of closed manifolds.

1.4. Legendrian and transversal curves. A curve $\Gamma \subset M$ is called Legendrian if it is tangent to the contact structure. If the curve is transversal to $\xi$ then it is called transversal. 
It is well known that sufficiently small neighborhoods of Legendrian (transversal) curves are contactomorphic via a contactomorphism that maps the first curve onto the second; thus the contact structure in the neighborhood of a curve is somewhat easier to study than the contact structure in the neighborhood of a surface.

At the same time there are purely contact obstructions to a global contactomorphism which takes a given transversal curve to another in the case when the curves in question are homologous to 0. It is defined as follows:

Let $\Gamma$ be a transversal curve, which is spanned by an embedded surface $F$; that is, $\Gamma=\partial F$. We can choose a non-vanishing vector field $\nu$ which is tangent to $\xi_{\mid F}$. Since $\Gamma$ is transversal, $\nu$ must be transversal to $\Gamma$, so we can push $\Gamma$ along $\nu$ and obtain a curve $\Gamma^{\prime}$ disjoint from $\Gamma$. We can now take the intersection number of $\Gamma^{\prime}$ and $F$. This number is called the self-linking number of $\Gamma$; it is traditionally denoted by $l(\Gamma)$.

It is important to note that the intersection number of $\Gamma^{\prime}$ and $F$ depends on their orientation. Transversal curves have a natural orientation given by a co-orientation of the contact structure (that is, a vector $v \in T_{x}(\Gamma)$ gives the proper orientation of $\Gamma$ if $\alpha(v)>0$ ). The curve $\Gamma^{\prime}$ inherits this orientation from $\Gamma$. The orientation of the surface $F$ can also be induced from the orientation of $\Gamma$. The orientation of $F$ which is commonly accepted induces an orientation of the characteristic foliation such that all the leaves of $F_{\xi}$ exit through the boundary.

Bennequin showed that if we consider the 3 -sphere with the standard contact structure, then the self-linking number satisfies the following inequality:

$$
l(\Gamma) \leq-\chi(F)
$$

where $\chi(F)$ is the Euler number of $F$.

Eliashberg showed that this inequality is satisfied on all tight manifolds. He also showed that if the characteristic foliation of the spanning surface has only elliptic and hyperbolic singularities, then the self-linking number can be calculated by adding the number of negative elliptic singularities to the number of positive hyperbolic singularities and subtracting from that the number of negative elliptic singularities and the number of positive hyperbolic singularities of $F_{\xi}$. This implies, for example, that the self-linking number of a curve which spans a disk (the un-knot) is always a negative odd number. See [El4], [Be] for a more complete treatment.

1.5. Main results. The following is a description of the main results of this paper.

Definition. A torus $T$ will be called normal if $T_{\xi}$ has no singularities and no Reeb components (section 3).

We shall show in Lemma 3.6 that any torus in a tight manifold can be made normal via a $\mathcal{C}^{0}$ small deformation.

If $T$ is a (normal) boundary of a solid torus $K$, then $\operatorname{conj}_{\Phi}(K)$ will represent the conjugacy class of the Poincaré return map of a transversal meridian, the rotation number of $K$, denoted by $\mathrm{r}(K) \in \mathbb{R} / 2 \pi \mathbb{Z}$, will be the rotation number of this Poincaré return map, and the self-linking number $1(K) \in \mathbb{Z}$ will be the self-linking number of the meridian (section 4).

It is not difficult to see that the quantities defined serve as contact invariants of closed solid tori which have normal boundary. The next theorem shows that, under certain conditions, these invariants give a complete classification of a tight contact structure on a closed solid torus. 
Theorem 5.4. Let $M_{1}, M_{2}$ be solid tori with tight contact structures and normal boundaries. Assume $\mathrm{l}\left(M_{1}\right)=\mathrm{l}\left(M_{2}\right)=-1$; then the two tori are contactomorphic iff $\operatorname{conj}_{\Phi}\left(M_{1}\right)=\operatorname{conj}_{\Phi}\left(M_{2}\right)$. Furthermore, if a diffeomorphism of the boundaries maps meridians to meridians and preserves the characteristic foliation, then it can be extended to a contactomorphism of the two tori.

While the above result dealt with tight contact structure on a closed solid torus, the following result deals with contact invariants of an interior of a tight solid torus.

Theorem 6.1. If $K, K^{\prime}$ are two solid tori with normal boundaries such that there exists a contactomorphism $\phi: \stackrel{\circ}{K} \rightarrow \stackrel{\circ}{K^{\prime}}$ then $\mathrm{r}(K)=\mathrm{r}\left(K^{\prime}\right)$ and $\mathrm{l}(K)=\mathrm{l}\left(K^{\prime}\right)$.

In Lemma 6.5 we construct two solid tori with normal boundaries and noncontactomorphic interiors which have the same self-linking number and the same rotation number. This shows that these two invariants do not fix the diffeomorphism type of a tight contact structure on an open solid torus.

Finally, in the last section (section 9), we construct more examples of tight contact structures on solid tori. In particular we prove the following:

Theorem 9.1. For any $j \in \mathbb{Z}^{>0}$ there exists a solid torus $T_{j} \subset\left(\mathbb{S}^{3}, \xi\right)$ (where $\xi$ is the standard, tight contact structure on $\mathbb{S}^{3}$ ) such that

(1) For some finite-sheeted cover $\psi: \widetilde{T}_{j} \rightarrow T_{j}$ the contact structure $\psi^{*}(\xi)$ is overtwisted;

(2) $\mathrm{l}\left(T_{j}\right)=-2 j-1$.

As we already mentioned the self-linking number of any un-knot is always odd; therefore, we produce an example of a tight torus with an overtwisted cover for every self-linking number, except the trivial one. In the trivial case, the results of the previous sections imply that this can never happen.

We also show

Theorem 9.3. There exists a tight contact structure $\hat{\xi}$ on $\mathbb{K}=\mathbb{R}^{2} \times \mathbb{S}^{1}$ which is not contactomorphic to any subset of $\left(\mathbb{S}^{3}, \xi\right)$ where $\xi$ is tight.

\section{LOCAL QUESTIONS}

This section shall be devoted to the study of the local properties of a contact structure. In particular we shall be interested in giving the sufficient conditions under which a (smooth) map between two sets can be extended to a contactomorphism of their neighborhoods. We start with a definition.

Definition 2.1. Two sets $S_{i} \subset\left(M_{i}, \xi_{i}\right)$ will be called contactly equivalent if there exist neighborhoods $U_{i} \subset M_{i}$ of $S_{i}$, and a contactomorphism $\phi: U_{1} \rightarrow U_{2}$ such that $\phi\left(S_{1}\right)=S_{2}$.

A map $f: S_{1} \rightarrow S_{2}$ will be called c-extendable if it is possible to choose $U_{i}, \phi$ so that $\phi_{\mid S_{1}}=f$. In that case $\phi$ will be said to extend $f$.

The main tool for investigating the properties of c-extendable diffeomorphisms between sets is based on the following lemma due to Moser [Mo]:

Lemma 2.2. Let $\xi_{t}$ be a smooth family of contact structures on a manifold $M$. Assume that there exists a family $\phi_{t}$ of smooth diffeomorphisms of $M$ generated by 
the vector field $X_{t}$, such that $X_{t} \in \xi_{t}$ and there exists a smooth family of 1-forms $\alpha_{t} \in \mathcal{A}_{\xi_{t}}^{+}$such that:

$$
\left.X_{t}\right\lrcorner d \alpha_{t \mid \xi_{t}}=\left(-\frac{\partial}{\partial t} \alpha_{t}\right)_{\mid \xi_{t}}
$$

Then $\phi_{t}^{*} \mathcal{A}_{\xi_{t}}^{+}=\mathcal{A}_{\xi_{0}}^{+}$.

Furthermore, for any smooth family $\alpha_{t} \in \mathcal{A}_{\xi_{t}}^{+}$there exists a unique (smooth) vector field $X_{t}$ which satisfies the above equation.

In the case where the manifold $M$ is such that it is possible to guarantee the integrability of all vector fields, then this lemma immediately implies that any two contact structures on $M$ which are homotopic in the class of contact structures are isotopic (this is known as the Gray's Theorem [Gr]).

Another immediate corollary of the lemma is the following very useful result:

Lemma 2.3. Let $\xi_{0}, \xi_{1}$ be two contact structures on a manifold $M$. Let $S \subset M$ be a subset of $M$ such that $\left(\xi_{0}\right)_{\mid S}=\left(\xi_{1}\right)_{\mid S}$. Then there exist neighborhoods $U_{i}$ of $S$ and a diffeomorphism $\phi: U_{0} \rightarrow U_{1}$ such that $\phi^{*}\left(\xi_{0}\right)=\xi_{1}$ and $\phi(x)=x$ for all $x \in S$.

In many cases, it is possible to give even weaker conditions under which the conclusion of Lemma 2.3 will still hold. It is well known, for example, that the characteristic foliation of the surface determines, to a large extent, the germ of the contact structure near that surface. This was shown, in particular, by Giroux.

Note that the same argument applies to a surface with boundary as long as the two contact structures are known to coincide in a neighborhood of the boundary. It is also easy to generalize the above proposition to the setting of c-extendable diffeomorphisms; in that setting, however, the case of surfaces with boundary becomes rather complicated. In order to show that a diffeomorphism $f: F_{1} \rightarrow F_{2}$, between surfaces with boundary, is c-extendable, it is necessary to show that the function $f_{\mid \partial F_{1}}$ is already c-extendable and that the extending contactomorphism $\phi$ can be chosen so that $\phi_{F_{1} \cap U}=f$ for some neighborhood $U$ of $\partial F_{1}$. This is not always easy to show in general, but in the case when $\partial F_{i}$ are transversal this condition can be easily fulfilled.

Thus in the language of c-extendable diffeomorphisms we have:

Proposition 2.6. A diffeomorphism between two closed surfaces or between two surfaces with transversal boundary is c-extendable iff it preserves the characteristic foliation.

We now proceed to study the structure of the contact structure in a neighborhood of 2-dimensional complex which is no longer a smooth surface. The complexity of the next proposition is entirely in the statement.

Proposition 2.7. Let $F_{i}, G_{i} \subset\left(M_{i}, \xi_{i}\right)$ be surfaces which satisfy the following transversality conditions:

(1) $\partial F_{i} \pitchfork \xi_{i}$ and $\partial G_{i} \pitchfork \xi_{i}$ (the boundaries are transversal to the contact structure);

(2) $F_{i} \pitchfork G_{i}$ (the surfaces are transversal to each other);

(3) the intersection of the two surfaces is a finite union of closed transversal curves; 
(4) each connected component of the intersection that intersects with the boundary of one of the surfaces is contained in the boundary of that surface.

Further assume that there exist diffeomorphisms $f: F_{1} \rightarrow F_{2}$ and $g: G_{1} \rightarrow G_{2}$ such that $f$ and $g$ preserve the characteristic foliation, and $f_{\mid F_{1} \cap G_{1}}=g_{\mid F_{1} \cap G_{1}}(f, g$ coincide on the intersection).

Then there exist $U \subset M_{1}$, a neighborhood of $F_{1} \cup G_{1}$ and a contactomorphic inclusion $\phi: U \rightarrow M_{2}$ such that $\phi$ extends both $f$ and $g$.

Proof. Lemma 2.3 implies that is sufficient to extend the functions $f$ and $g$ by a common diffeomorphism of the neighborhood of $F_{1} \cup G_{1}$.

Note that any such extension of this type will take the contact plane to the contact plane at the points of intersection of the two surfaces. We can further make sure that this happens on the surfaces near the intersection set as well. We now apply the previous argument.

The motivation behind the following definitions should be pretty clear.

Definition 2.8. A family of smooth surfaces $\left\{F_{j}\right\}^{j \leq n}$ in a contact manifold $M$ will be said to have transversal corners if any pair of surfaces $F, F^{\prime} \in\left\{F_{j}\right\}$ satisfy the conditions (1)-(4) imposed on $F_{1}$ and $F_{1}^{\prime}$ in Proposition 2.7.

A piecewise smooth surface $F \subset M$ will be said to have singularities of transversal corner type if there exists a family with transversal corners $\left\{F_{j}\right\}^{j \leq n}$ such that $F=\bigcup_{j}^{n} F_{j}$.

A manifold with a piecewise smooth boundary will be said to have transversal corners if the boundary has singularities of transversal corner type.

There are three types of transversal corners singularities.

(1) A curve which is a boundary component of two of the surfaces;

(2) A curve which belongs to the interior of one surface and to the boundary of another;

(3) A curve which belongs to the interior of two surfaces.

On a surface with transversal corners only type (1) can occur. The next lemma shows that a piecewise smooth surface with transversal corner singularities can be approximated quite well by smooth surfaces.

Lemma 2.9. Let $F \subset M$ be a piecewise smooth surface with transversal corner singularities. Then there exists a $\mathcal{C}^{0}$ small, piecewise smooth isotopy $h_{t}: M \rightarrow M$ such that

(1) $h_{0}=$ Id;

(2) $h_{t}$ is supported on an arbitrarily small tubular neighborhood $U \subset M$ of the singularities of $F$ (i.e. near the "corners");

(3) $h_{1}(F)$ is smooth;

(4) any two points $x, y \in F \backslash U$ which could be connected by a piecewise smooth Legendrian curve in $F$ lie on the same leaf of the characteristic foliation of $h_{1}(F)$

(5) $\left(h_{1}(F)\right)_{x} i$ has no new singularities.

What this lemma is saying is that a piecewise smooth surface with transversal corner singularities can be smoothed in such a way that the characteristic foliation of the smoothed surface is homeomorphic to the characteristic foliation of the original surface. 

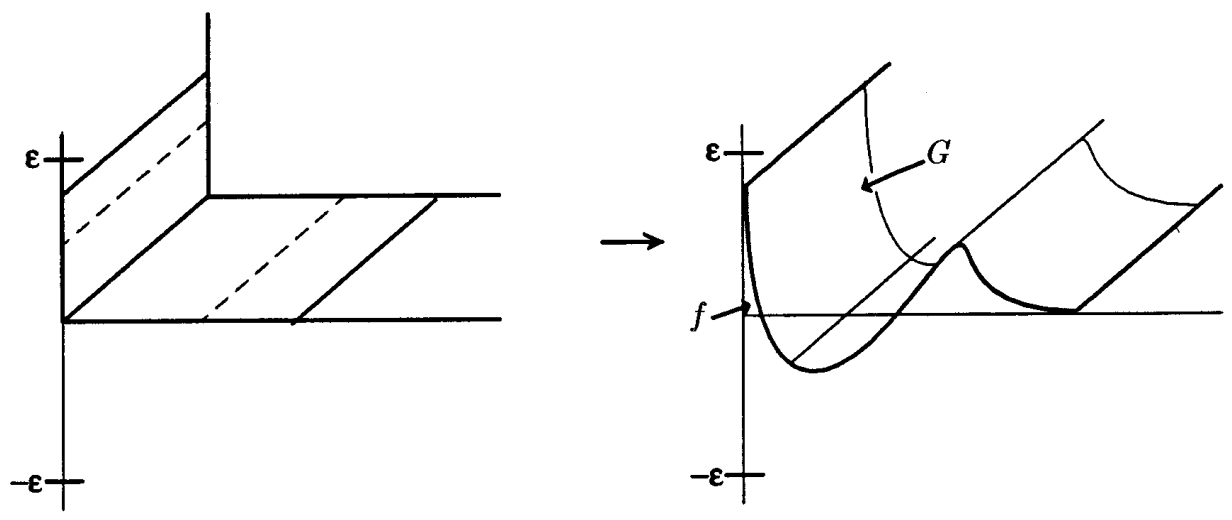

FIgURE 1. Smoothing out transversal corners singularities

Proof. By the definition of transversal corners $F=\bigcup_{j}^{n} F_{j}$ where $F_{j}$ are smooth surfaces with boundary. The set of singularities of $F$ consists of a finite number of transversal curves.

Choose one such curve $\Gamma$. $\Gamma$ is a connected component of the boundary of two smooth surfaces $F$ and $F^{\prime}$. The characteristic foliation of each of these surfaces has no singularities in some neighborhood of $\Gamma$.

Consider $\widehat{\mathbb{T}}=\mathbb{R}^{3} /\{z \rightarrow z+1\}$ with the contact structure defined by $d z=$ $y d x$. Proposition 2.7 implies that there exist a tubular neighborhood of $\Gamma$ and a contactomorphism $\phi: U \rightarrow \widehat{\mathbb{T}}$ such that

(1) $\phi(\Gamma)=\{x=y=0\} \subset \widehat{\mathbb{T}}$;

(2) $\phi(F \cap U)=\{x=0, y \in[0,1]\} \subset \widehat{\mathbb{T}}$;

(3) $\phi\left(F^{\prime} \cap U\right)=\{x \in[0,1], y=0\} \subset \widehat{\mathbb{T}}$.

Consider a function $f(x):[0,1] \rightarrow \mathbb{R}$ (see Figure 1 ) such that

(1) $\frac{d^{n} f(0)}{d x^{n}}=\infty$, for $n=1, \ldots, \infty$;

(2) $\frac{d^{n} f(\varepsilon)}{d x^{n}}=0$, for $n=1, \ldots, \infty$;

(3) $f(0)>0$ and $f_{\mid[\varepsilon, 1]}=0$;

(4) $\sup |f|<\varepsilon$;

(5) $\int_{0}^{1} f(x) d x=0$.

Let $G=\{x \in[0,1], y=f(x)\} \cup\{x=0, y \in[f(0), 1]\} \subset \widehat{\mathbb{T}}$; this is a smooth surface which is quite clearly homotopic to $\{x=0, y \in[0,1]\} \cup\{x \in[0,1], y=0\}$.

A simple calculation shows that the characteristic foliation of the surface $\{y=f(x, z)\}$ is given by the vector field $\frac{\partial}{\partial x}+f(x, z) \frac{\partial}{\partial z}+\left(f(x, z) f_{z}+f_{x}\right) \frac{\partial}{\partial y}$. This means that the leaves of the characteristic foliation connect the point $(1,0, z)$ with the point $(0,1, z)$ (points on the boundary which have the same $z$ coordinates), just as the piecewise smooth Legendrian curves which lie on $\{x=0, y \in[0,1]\} \cup$ $\{x \in[0,1], y=0\}$. Thus, the surface $F \backslash U \cup \phi^{-1}(G)$ will satisfy all the necessary conditions (assuming that $\varepsilon$ is small enough).

We can now apply the same procedure to all the other curves in the singular set to obtain the required smooth surface.

We finish this section with a classification of tight contact structures on balls with transversal corners. 
Lemma 2.10 (Classification of balls with transversal corners). Let $M_{1}$ and $M_{2}$ be tight contact balls with transversal corners which have boundaries which are contactly equivalent. Then the manifolds themselves are contactomorphic. Furthermore, any c-extendable diffeomorphism of the boundaries extends to a contactomorphism of the whole manifold.

Proof. Given $f$, a c-extendable diffeomorphism between the boundaries of $M_{1}$ and $M_{2}$, let $\phi: U_{1} \rightarrow U_{2}$ be the contactomorphism which extends $f$ to a neighborhood of the boundary. Now choose a smooth sphere $S \subset U_{1}$ which is a boundary of a ball $B \subset M$ so that $U_{1} \cup B=M_{1}$. According to a classification theorem of Eliashberg ([El1] Theorem 1) there exists a contactomorphism between $B$ and the ball in $M_{2}$ whose boundary is $\phi(S)$ and this contactomorphism can be chosen to extend $\phi$. This provides the necessary contactomorphism.

\section{Deformations and Characteristic Foliations}

In this section we will discuss various results which allow us to simplify the characteristic foliation of surfaces embedded in a tight contact manifold by deforming these surfaces.

Throughout this section we shall assume that all surfaces lie in $M$ where $M$ is a 3-manifold with a tight contact structure $\xi$.

Definition 3.1. If $K$ is a closure of an open subset of an oriented surface $F$ and if $K$ has a smooth boundary, it is said to be positive (negative) if $\partial K$ is transversal to $F_{\xi}$ and all the leaves of $F_{\xi}$ exit (enter) $K$.

A cycle $\gamma$ of the characteristic foliation is said to be positive (negative) if any neighborhood $U$ of $\gamma$ contains a positive (negative) set which contains $\gamma$.

A basin $B(K)_{F}$ is defined to be the union of all the non-singular leaves of $F_{\xi}$ which intersect $K$. If $K$ is a positive (negative) set then, $B(K)_{F}$ is the set of points of $F$ which can be reached from $K$ (can reach $K$ ) along the non-singular trajectories of $F_{\xi}$.

If $F_{\xi}$ is generic, then a point on the boundary of a basin of a positive (negative) set will belong to one of the following 4 types of sets:

(1) The boundary of the surface $F$;

(2) The limit cycles of $F_{\xi}$;

(3) The elliptic and hyperbolic points of $F_{\xi}$ (i.e. the singularities);

(4) The separatrixes of $F_{\xi}$.

If the boundary of the basin contains only the last three types of points, then it will be called semistandard.

The main tool for simplifying the characteristic foliation of a surface is the socalled elimination lemma ([Gi1], [El1]) which states that a surface with a characteristic foliation which contains hyperbolic and elliptic singularities of the same sign which lie on the same leaf can be killed via a $\mathcal{C}^{0}$ small perturbation of the surface which is fixed on the leaf and supported in a neighborhood of that leaf.

The elimination lemma implies that (for tight contact structure) the characteristic foliation cannot contain contractable leaves which pass through singularities of the same sign, since it is possible to make such a leaf into a contractable cycle. Eliashberg used this fact to obtain a very strong statement about the combinatorics of the characteristic foliation ([El1] Proposition 3.5.1 and Lemma 3.7.1 or [El4] Lemma 4.3.2). 
Lemma 3.2. If $K$ is a positive or a negative subset of a closed surface $F$ and if the basin $B=B(K)_{F}$ is semistandard, then either

(1) $\bar{B}=F \cong \mathbb{S}^{2}$

or

(2) the boundary of $B$ contains at least one hyperbolic point of the same sign as $K$.

The next few lemmas deal with the behavior of the characteristic foliation near a cycle.

Lemma 3.3. Let $\gamma \subset F$ be a closed, positive cycle of $F_{\xi}$. There exists a $\mathcal{C}^{0}$ small isotopy $h_{t}: M \rightarrow M$ fixed on $\gamma$ and supported in an arbitrarily small neighborhood of $\gamma$ such that

(1) $h_{0}=\mathrm{Id}$;

(2) $h_{1}(F)_{\xi}$ has no new singularities;

(3) $h_{1}(F)_{\xi}$ has the same number of cycles as $F_{\xi}$;

(4) the orientation of $\gamma$ as a leaf of $h_{1}(F)_{\xi}$ is opposite to its orientation as a leaf of $F_{\xi}$.

Before starting the proof we remind the reader that the only smooth invariant of a nonsingular foliation near a non-degenerate closed leaf is the germ of the Poincaré return map. A closed cycle is non-degenerate if the eigenvalue of the linearization of the Poincaré return map is not equal to one.

Proof. Consider $\mathbb{R}^{3}=\{(\theta, r, z)\}$ with the contact structure induced by the one form $\cos (2 \pi r) d z-r \sin (2 \pi r) d \theta$. It is easy to see that the curve $\tilde{\gamma}=\{r=1, z=0\} \subset \mathbb{R}^{3}$ is Legendrian and that the cylinder $\{r=1\}$ is foliated by translates of that curve.

Let $\gamma$ be the limit cycle of $F_{\xi}$. The characteristic foliation near $\gamma$ is non-singular and the cycle is positive; this means that the eigenvalue must be greater than one.

It is also not difficult (but very tedious) to construct a smooth function $\{f(r)\}$ such that

(1) $f_{\mu}(1)=0$

(2) $f_{\mu}(\theta, r)>0$ for all $r>1$ and $f_{\mu}(\theta, r)<0$ for all $r<1$;

(3) the characteristic foliation of the surface $\Gamma_{f}=\{z=f(r)\} \subset \mathbb{R}^{3}$ has $\tilde{\gamma}$ as a positive limit cycle;

(4) there exist some tubular neighborhood $U$ of the curve $\gamma$ and a contactomorphism $\psi: U \rightarrow \mathbb{R}^{3}$ which takes $\gamma$ to $\tilde{\gamma}$ and takes $F \cap U$ into $\Gamma_{f}$.

To satisfy property (4) it is simply necessary to make sure that the germ of the Poincaré return map of $F_{\xi}$ near $\gamma$ is the same as the germ of Poincaré return map of the characteristic foliation of $\Gamma_{f}$ near $\tilde{\gamma}$.

A simple calculation shows that the contact plane at a point $x=(\theta, 1,0) \in \tilde{\gamma}$ is spanned by the vectors $\frac{\partial}{\partial r}(x)$ and $\frac{\partial}{\partial \theta}(x)$. Since $\tilde{\gamma} \subset \psi(F \cap U)$, the vector $\frac{\partial}{\partial r}(x)$ must be transversal to the surface. Therefore, the orientation of $\tilde{\gamma}$ induced by $\psi(F \cap U)$ depends on whether $\frac{\partial}{\partial r}$ agrees or disagrees with the co-orientation of the surface.

Choose now a smooth function $g$, such that

(1) $g(r)=f(r)$ for all $|r|>\varepsilon_{1}$ for some sufficiently small $0<\varepsilon_{1}$;

(2) $g(r)=-f(r)$ for all $|r|<\varepsilon_{2}$ for some $0<\varepsilon_{2}<\varepsilon_{1}$.

Let $\Gamma_{g}=\{z=g(r)\} \subset \mathbb{R}^{3}$. The co-orientation induced by $\frac{\partial}{\partial r}$ on the graph of $\Gamma_{g}$ is opposite to that of the graph of $\Gamma_{f}$. The only closed leaf of the characteristic foliation of $\Gamma_{g} \cap U$ is $\tilde{\gamma}$ because the characteristic foliation of $\Gamma_{g}$ is invariant with 
respect to rotations around the $z$ axis and the only Legendrian curves with that property near $\tilde{\gamma}$ lie on a cylinder $\{r=1\}$. The characteristic foliation of $\left(\Gamma_{g} \cap U\right)$ has no singularities for the same reason.

Clearly we can deform $\Gamma_{f}$ to $\Gamma_{g}$ via an isotopy $\tilde{h}_{t}$ which is supported inside $W \subset \psi(U)$ and fixed on $\tilde{\gamma}$. The size of $\tilde{h}_{t}$ depends on $\varepsilon_{1}, \varepsilon_{2}$; therefore, the isotopy $\psi^{-1}\left(\tilde{h}_{t}\right)$ can be chosen to be arbitrarily small.

Note that the above argument can be applied to the negative cycles as well.

The next two lemmas can be proved by similar arguments.

Lemma 3.4. Let $F_{i}$ be two surfaces. Assume $\gamma_{i} \subset F_{i}$ are cycles of the respective characteristic foliations. Then there exists a $\mathcal{C}^{0}$ small isotopy $h_{t}: M \rightarrow M, t \in$ $[0,1]$, and a $U_{i}$ a tubular neighborhood of $\gamma_{i}$ such that

(1) $h_{0}=\mathrm{Id}$;

(2) $h_{t}$ is fixed on $\gamma_{1}$ and outside $U_{1}$;

(3) $\left(h_{1}(F) \cap U_{1}\right)_{\xi}$ is contactly equivalent to $\left(F_{2} \cap U_{2}\right)_{\xi}$.

To prove this lemma we construct an isotopy between the graphs of the functions that represent the two cycles.

Lemma 3.5. Let $F$ be a surface. Assume $\gamma \subset F$ is a cycle of $F_{\xi}$. Then there exist $a \mathcal{C}^{0}$ small isotopy $h_{t}: M \rightarrow M, t \in[0,1]$, and $U$, a tubular neighborhood of $\gamma$ such that

(1) $h_{0}=\mathrm{Id}$;

(2) $h_{t}$ is fixed on $\gamma$ and outside $U$;

(3) $\left(h_{1}(F) \cap U\right)_{\xi}$ has two more cycles than before.

To prove this lemma we deform the graph of the functions that represent the cycle and make it intersect the cylinder $\{r=1\} \subset \mathbb{R}^{3}$. In this case it is easier to think of a graph of a function in the $z$ and $\theta$ variables (see Figure 2).

Before stating the next lemma, we remind the reader that a Reeb component of a foliation on a surface is an annulus inside that surface bounded by integral curves of the foliation such that the interior of that annulus is homeomorphic to the annulus $A=(x, y)=(0,1) \times \mathbb{R} / \mathbb{Z}$ with the foliation given by the curves $c_{a}(t)=\left(t,\left(\frac{1}{t}+\frac{1}{t+1}+a\right)\right)$ for $t \in(0,1), a \in \mathbb{R}$.

Note that the two boundary curves of such an annulus must have opposite orientations.

Lemma 3.6. If $M$ is a manifold with tight contact structure $\xi$, then any closed surface of positive genus $F \subset M$ can be deformed by a $\mathcal{C}^{0}$ small isotopy $h_{t}: M \rightarrow M$ so that $h_{1}(F)_{\xi}$ has only hyperbolic singularities and no Reeb components.

Proof. By deforming the surface slightly we can assume that the characteristic foliation is generic. That means

(1) all the singularities are either hyperbolic or elliptic;

(2) there are no hyperbolic-hyperbolic connections;

(3) all the non-singular closed leaves are either positive or negative.

Since any basin on a closed surface is semistandard Lemma 3.2 implies that any elliptic singularity of $F_{\xi}$ is always connected with a hyperbolic singularity; thus, by the elimination lemma all the elliptic points can be killed via a $\mathcal{C}^{0}$ small isotopy. 


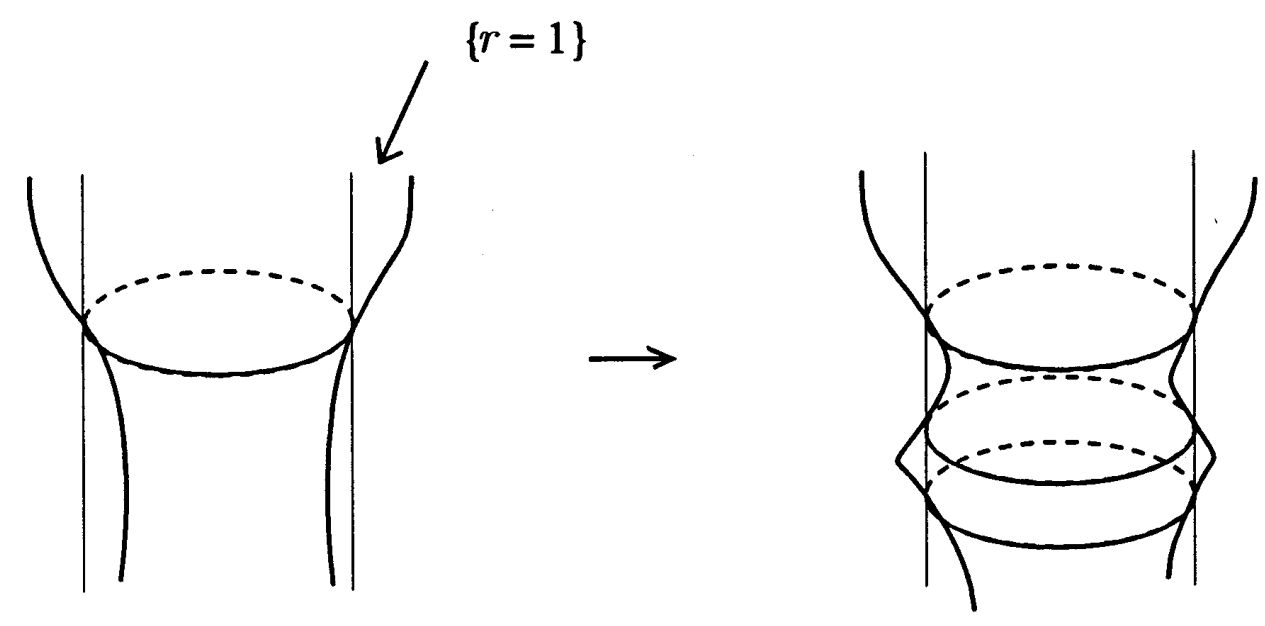

FiguRE 2. Creating new cycles

The existence of a Reeb component implies that there are limit cycles oriented in opposite directions, but Lemma 3.3 implies that we can deform the surface in such a way that all the limit cycles are oriented in the same way.

Note that if the surface is a torus, we have shown that we can kill all the singularities.

Definition 3.7. A closed surface will be called normal if its characteristic foliation has no Reeb components and no elliptic singularities.

Another result which we shall find useful is a result proved by Eliashberg (Lemma 3.6.1 in [El1]).

Lemma 3.8. If $M$ is a manifold with tight contact structure $\xi$, then any disk $D \subset$ $M$ such that $\partial D$ is transversal can be deformed by a $\mathcal{C}^{0}$ small isotopy $h_{t}: M \rightarrow M$ so that $h_{1}(D)_{\xi}$ has no elliptic and hyperbolic singularities of the same sign. Furthermore, the isotopy can be chosen to be fixed in a sufficiently small neighborhood of $\partial D$.

This result together with some other results of Eliashberg also shows that disks with transversal boundaries are almost contactly equivalent; specifically we can prove:

Lemma 3.9. Let $\Gamma$ be a transversal curve such that $\Gamma=\partial D$ for some disk $D$. Let $D^{\prime}$ be another disk with transversal boundary such that $l\left(\partial D^{\prime}\right)=l(\Gamma)$. For any, arbitrarily small, tubular neighborhood $U$ of $D$ there exists a disk $\widehat{D} \subset U$ such that

(1) $\widehat{D}_{\xi}$ is diffeomorphic to $D_{\xi}^{\prime}$, and

(2) $\partial \widehat{D}=\Gamma$.

Proof. The classification of contact balls ([El1]) implies that $U$ is contactomorphic to any tubular neighborhood of the disk $D^{\prime}$. Let $\psi: V \rightarrow U$ be such a contactomorphism. Then the disk $\psi\left(D^{\prime}\right)$ satisfies the condition (1). Since $\Gamma$ and $\partial\left(\psi\left(D^{\prime}\right)\right)$ are transversal curves with the same self-linking number and these curves are both spanned by disks, there exists a contactomorphism $\phi: U \rightarrow U$ which maps the 
curve $\partial\left(\psi\left(D^{\prime}\right)\right)$ to the curve $\Gamma$ (see [El4]). The disk $\phi\left(\psi\left(D^{\prime}\right)\right)$ satisfies the two conditions.

We shall later improve this result (see Lemma 4.6), which shall show that the $\widehat{D}$ can be deformed to coincide with $D$ near the boundary in a way that does not change its characteristic foliation.

The situation of other surfaces with boundary is, unfortunately, much more complex. It is possible, for example, to construct an annulus $A$ with transversal boundary and such that $l(\partial A)=0$; nevertheless, the singularities of $A_{\xi}$ are stable with respect to $\mathcal{C}^{0}$ small perturbations. The lemma below provides sufficient conditions which allow us to avoid this situation.

Lemma 3.10. If $M$ is a manifold with tight contact structure $\xi$, and $A \subset M$ is an annulus such that

(1) $\partial A=\Gamma \cup \Gamma^{\prime}$ where $\Gamma$ and $\Gamma^{\prime}$ are transversal curves;

(2) $l(\Gamma)=l\left(\Gamma^{\prime}\right)=-1$;

(3) there exists a disk $D \subset M$ such that $D \cap A=\Gamma$;

(4) $[\Gamma]=\left[\Gamma^{\prime}\right]$ in $H_{1}(A)$ (the orientations of $\Gamma$ and $\Gamma^{\prime}$ match up),

then $A$ can be deformed by a $\mathcal{C}^{0}$ small isotopy $h_{t}: M \rightarrow M$ so that $h_{1}(A)_{\xi}$ has no singularities (i.e. $\left.h_{1}(A) \pitchfork \xi\right)$.

Proof. Without loss of generality we can assume that $D \pitchfork A$. In that case we have $D \cup A$ is a piecewise smooth disk with transversal corners. We can also assume that $D_{\xi}$ has no negative elliptic points and no positive hyperbolic points, since we can deform $D$ away from the boundary to kill all these by Lemma 3.8 (assuming we chose the proper orientation). Now the self-linking number of the boundary is equal to the total index of rotation of the negative singularities minus the total index of rotation of the positive singularities (introduction, section 1.4). Therefore, since $l(\partial D)=-1$, the characteristic foliation of $D$ must have only one positive elliptic singularity and no others. Call that point $e$.

By condition (2), the number of elliptic and hyperbolic singularities of the same sign on $A_{\xi}$ should be equal. We can also assume that $A_{\xi}$ has no singularities of the same sign connected by a leaf, since we could use the elimination lemma to kill these. We now consider the characteristic foliation on the piecewise smooth disk $A \cup D$.

If it has a positive hyperbolic singularity $h$, then $h \in A$. Note that the incoming separatrixes of $h$ must come from an elliptic singularity which is a source and, therefore, positive. Since we have already assumed that no positive elliptic singularities of $A_{\xi}$ can be connected to $h$, both the incoming separatrixes must come from $e$. But, if that were the case, we could first use Lemma 2.9, to smooth out the surface without destroying these connections, and then we could use the elimination lemma to produce a cycle. This would contradict the assumption that the contact structure is tight. Thus there cannot be any positive hyperbolic or elliptic singularities.

Similar reasoning shows that there can be no negative singularities either.

We finish by mentioning a well known result which allows us to create singular points and to make the characteristic foliation of a surface more complicated (Lemma 3.3.2 [El1], [Gi1]). 
Lemma 3.11 (The creation of singularities). Let $p, q \in F$ be two points which are connected by a non-singular leaf of $F_{\xi}$. Then there exists a smooth $\mathcal{C}^{0}$ small isotopy $h_{t}: M \rightarrow M, t \in[0,1]$, such that

(1) $h_{0}=\mathrm{Id}$;

(2) $h_{t}$ is fixed on $\gamma$ and outside some neighborhood $U$ of $\gamma$, where $\gamma$ is the leaf that connects $p$ to $q$;

(3) $\left(h_{1}(F)\right)_{\xi}$ has exactly two new singularities, a hyperbolic one at $q$ and an elliptic one at $p$.

These singularities can be made either both positive or both negative.

\section{PROPERTIES OF NORMAL FOLIATIONS ON TORI AND ROTATION NUMBERS}

The purpose of this section is to understand more deeply the nature of the behavior of a tight contact structure near a normal torus and to define some contact invariant solid tori with normal boundaries. We will also construct a contact model for the neighborhood of the torus $T$.

The situation we will be considering will be the following:

1. $M$ will be a 3 -manifold with contact structure $\xi$;

2. $T \subset M$ will be a normal torus;

3. By $\nu$ we will represent the vector field that induces the characteristic foliation on $T$;

4. $H=H_{1}(T, \mathbb{Z})$;

5. All curves will be assumed to be smooth and to lie in $T$;

6. $\mathbb{T}$ will represent $\mathbb{R}^{3} /\{z \mapsto z+1\}$ with the contact structure given by the kernel of $d z+r^{2} d \theta$.

Definition 4.1. A curve in $T$ will be called super-transversal if it is transversal to $\nu$ and intersects every leaf of the characteristic foliation at least once.

A homology class in $H$ will be called Legendrian if it can be represented by a cycle of the characteristic foliation.

Note that:

(1) A transversal curve which represents a non-Legendrian class is always supertransversal.

(2) There can be at most one Legendrian homology class (up to orientation).

(3) Given any (connected embedded) curve $\gamma \in T$ such that $[\gamma] \in H$ is not a Legendrian class, there exists a curve $\Gamma$ in $T$ such that $\Gamma \in[\gamma]$ and $\Gamma$ is supertransversal; furthermore, the choice of such a curve is unique, up to an isotopy which preserves the foliation. In this case the orientation of the transversal representative is given by the class orientation of the curve $\gamma$ so the transversal curve can be either positively or negatively oriented with respect to the contact structure.

This allows us to define the following quantities:

Definition 4.2. Let $\Gamma \subset T$ be a positively oriented super-transversal curve in $T$, and let $[\Gamma] \in H_{1}(T)$ be a cycle such that $\Gamma \in[\Gamma]$.

(1) $\Phi_{T, \Gamma}$ will represent the Poincaré return map of $\Gamma$ with respect to the $T_{\xi}$;

(2) $\operatorname{conj}_{\Phi,[\Gamma]}(T)$ will represent the conjugacy class of $\gamma^{-1} \circ \Phi_{T, \Gamma} \circ \gamma: \mathbb{S}^{1} \rightarrow \mathbb{S}^{1}$, where $\gamma$ is a parametrization of $\Gamma$.

If $T$ is a boundary of a solid torus $K$ and $\Gamma$ is the meridian, then

(3) $\Gamma$ will be called an essential curve of $T$; 
(4) $\operatorname{conj}_{\Phi}(K)=\operatorname{conj}_{\Phi,[\Gamma]}(T)$ will represent the conjugacy class of the Poincaré return map of the meridian with respect to the characteristic foliation of the boundary;

(5) The self-linking number of $K$ will be defined as $\mathrm{l}(K)=l(\Gamma)$.

If $T_{i}=\partial K_{i}$ where $K_{i}$ are solid tori then a map between the tori can be extended to the interior iff it takes the class of the meridian to the class of the meridian in homology. Thus two solid tori cannot be contactomorphic unless $1\left(K_{1}\right)=1\left(K_{2}\right)$.

At the same time the existence of a diffeomorphism $f: T_{1} \rightarrow T_{2}$ such that $f$ preserves the foliation and $f_{*}\left(\left[\Gamma_{1}\right]\right)=\left[\Gamma_{2}\right]$ is equivalent to the condition that $\operatorname{conj}_{\Phi,\left[\Gamma_{1}\right]}\left(T_{1}\right)=\operatorname{conj}_{\Phi,\left[\Gamma_{2}\right]}\left(T_{2}\right)([\mathrm{A} 2])$. So two solid tori which are contactomorphic must have $\operatorname{conj}_{\Phi}\left(K_{1}\right)=\operatorname{conj}_{\Phi}\left(K_{2}\right)$.

While $1(K)$ is an invariant that is easily computable, $\operatorname{conj}_{\Phi}(K)$ is not as easy because it is extremely difficult to compare conjugacy classes of diffeomorphisms of the circle in general. A well-known tool for studying this question is an invariant of conjugacy classes of diffeomorphisms of a circle called the rotation number.

Definition 4.3. The rotation number of a normal torus with respect to a homology class $[\Gamma]$ will be defined as $\mathrm{r}_{[\Gamma]}(T)=r(\Psi)$ where $\Psi \in \operatorname{conj}_{\Phi,[\Gamma]}(T)$ and $r(\Psi)$ is the rotation number of $\Psi$. The rotation number of a solid torus $K$ (with a normal boundary) will be defined as $\mathrm{r}(K)=\mathrm{r}_{[\Gamma]}(\partial K)$ where $\Gamma$ is the meridian.

Note that the rotation number of a diffeomorphism of a circle is only well defined $(\bmod 2 \pi)$. Therefore, when comparing two diffeomorphisms of the circle it is impossible to compare the size of the two rotation numbers, but only possible to say if the rotation numbers are equal. When considering the rotation number of a Poincaré return map of some fixed (super-transversal) curve $\Gamma \subset T$, it is possible to choose a consistent way of assigning a real value to the rotation number of the Poincaré return map by choosing a homology class $\beta \in H_{1}(T)$ such that $[\Gamma] \cdot \beta=1$ and requiring that the Poincaré return map of the foliation which consists of closed leaves in the class $\beta$ has the rotation number of 0 . A different choice of $\beta$ changes all the rotation numbers by same amount $\left(r_{2}=2 \pi \cdot \beta_{1} \cdot \beta_{2}+r_{1}\right)$. This means that if we have a transversal curve $\Gamma_{1} \subset T_{1}$ and another transversal curve $\Gamma_{2} \subset T_{2}$ then in order to compare the rotation numbers of two Poincaré return maps, it is enough to fix an isomorphism $\iota: H_{1}\left(T_{1}\right) \rightarrow H_{1}\left(T_{2}\right)$ such that $\iota\left(\left[\Gamma_{1}\right]\right)=\left[\Gamma_{2}\right]$. Thus, if there is some way to choose a canonical isomorphism between these two tori it is then possible to define the difference between the two rotation numbers as a real number.

We now prove a lemma which shall be used later to construct models of solid tori.

Lemma - Construction 4.4. Given a diffeomorphism of the circle $\Phi: \mathbb{S}^{1} \rightarrow$ $\mathbb{S}^{1}=\mathbb{R} / 2 \pi \mathbb{Z}$ which preserves orientation, there exists a twice periodic function $f: T^{2}=\mathbb{R}^{2} /(2 \pi \mathbb{Z} \oplus \mathbb{Z})=\{(\theta, z)\} \rightarrow \mathbb{R}^{>0}$ such that the points $(\theta, f(\theta, 0), 0) \in \mathbb{T}$ and $(\Phi(\theta), f(\Phi(\theta), 0), 0) \in \mathbb{T}$ lie on the same leaf of the characteristic foliation of the torus $T_{f}=\{r=f(\theta, z)\} \subset \mathbb{T}$.

Proof. Since $\Phi$ preserves orientation, it is isotopic to the identity. Let $F(\theta, z)$ : $\mathbb{S}^{1} \times[0,1] \rightarrow \mathbb{S}^{1}$ be a smooth isotopy such that

(1) $F(\theta, z)=\theta$, for $z \in[0, \varepsilon]$, and

(2) $F(\theta, z)=\Phi(\theta)$ for $z \in[1-\varepsilon, 1]$. 
Let $\nu^{\prime \prime}$ be a vector field on $\mathbb{S}^{1} \times[0,1]$ defined by $\frac{\partial}{\partial z}+\left(F_{z}(\theta, z)+2 k \pi\right) \frac{\partial}{\partial \theta}$ where $k$ is an integer such that $F_{z}+2 k>0$. Note that for $z>1-\varepsilon$ and for $z<\varepsilon, F_{z}(\theta, z)=0$; therefore, it is possible to define a smooth, periodic vector field $\nu^{\prime}$ on $\mathbb{S}^{1} \times \mathbb{R}$ by setting $\nu^{\prime}(\theta, z+n)=\nu^{\prime \prime}(\theta, z)$. This field, in turn, induces a field $\nu$ on $\mathbb{S}^{1} \times \mathbb{S}^{1}=$ $\mathbb{R}^{2} /(2 \pi Z \times Z)$. The integral curves of this vector field are given by the curves $c_{\theta}(t)=(F(\theta, t)+2 k \pi \cdot t ; t)$. In particular $c_{\theta}(0)=(F(\theta, 0)+2 k \pi ; 0)=(\Phi(\theta), 0)$, and $c_{\theta}(1)=(F(\theta, 1)+2 k \pi ; 1)=(\Phi(\theta), 0)$. Let $g(\theta, z)=\left(F_{z}(\theta, z)+2 k \pi\right)^{-1 / 2}$, and define $f: \mathbb{S}^{1} \times \mathbb{S}^{1} \rightarrow \mathbb{T}$ by $f(\theta, z)=(g(\theta, z), \theta, z)$. A simple calculation shows that $f_{*}(\nu) \in \xi$; thus $f$ is the required function.

We now show that all the tori of the type described above are actually normal.

Lemma 4.5. Let $f: T^{2}=\mathbb{R}^{2} /(2 \pi \mathbb{Z} \oplus \mathbb{Z})=(\theta, z) \rightarrow \mathbb{R}^{>0}$. Then the torus $T_{f}=$ $\{r=f(\theta, z)\} \subset \mathbb{R}^{3} /\{z \mapsto z+1\}$ is always a normal torus.

The proof is a straightforward calculation.

Before finishing this section we present an application of most of the results of the previous sections to give a stronger version of Lemma 3.9:

Lemma 4.6. Let $\Gamma$ be a transversal curve such that $\Gamma=\partial D$ for some disk $D \subset$ $M$ where $M$ is a tight contact manifold. Let $D^{\prime}$ be another disk with transversal boundary such that $l\left(\partial D^{\prime}\right)=l(\Gamma)$. For any, arbitrarily small, tubular neighborhood $U$ of $D$ there exists a disk $\widehat{D} \subset U$ such that

(1) $\widehat{D}_{\xi}$ is diffeomorphic to $D_{\xi}^{\prime}$;

(2) $\partial \widehat{D}=\Gamma$;

(3) $\widehat{D}$ coincides with $D$ near $\Gamma$.

Proof. Lemma 3.9 provides us with a disk $\widetilde{D}$ which satisfies conditions (1) and (2). We now proceed to deform this disk in such a way that it satisfies the condition (3). Proposition 2.6 implies that there exist a tubular neighborhood $U$ of $\Gamma$ and a contactomorphism $\phi: U \rightarrow \mathbb{T}$ such that $\phi(\Gamma)=\{r=\theta=0\}$ and $\phi(D \cap U)=$ $\{\theta=0, r<\varepsilon\}$. Since $\widetilde{D}$ is a smooth disk there exists a tubular neighborhood of $\Gamma, V \subset U$ such that the projection of $\phi(\widetilde{D} \cap V)$ onto the $z$ axis is non-singular. That means that there exists a curve $\Gamma^{\prime} \subset \phi(\widetilde{D} \cap V)$ for which this projection is non-singular. In other words $\Gamma^{\prime}=\{\theta=p(z), r=q(z)\} \subset \mathbb{T}$. Note that this makes $\Gamma^{\prime}$ a transversal curve.

Consider a function $f: T^{2} \rightarrow \mathbb{R}^{>0}$ such that

(1) $f(z, p(z))=r(z)$;

(2) $f_{z}>0$

(3) $K=\{r<f(z, \theta)\} \subset U$.

We know from Lemma 4.5 the torus $\phi^{-1}(\partial K)$ is normal. The condition (2) imposed on $f$ implies that the curve $\partial K \cap \phi(D)$ is transversal and so is the curve $\partial K \cap \phi(\widetilde{D})=$ $\Gamma^{\prime}$. Now the curve $\phi^{-1}(\partial K) \cap D$ is a boundary of a disk in $M$, so it cannot represent a Legendrian class in homology. The same holds for $\phi^{-1}(\partial K) \cap \widetilde{D}$, and these two curves obviously lie in the same homology class. Thus these two curves are transversely isotopic in $\phi^{-1}(\partial K)$. We can cover this transversal isotopy by a family of Hamiltonian contactomorphisms. So there exists a contactomorphism $\psi: M \backslash \phi^{-1}(K) \rightarrow M \backslash \phi^{-1}(K)$ such that $\psi\left(\phi^{-1}(\partial K) \cap \widetilde{D}\right)=\phi^{-1}(\partial K) \cap D$. Consider the disk $D \cap \phi^{-1}(\bar{K}) \cup \psi\left(\widetilde{D} \backslash \phi^{-1}(K)\right)$. This is a piecewise smooth disk 
with transversal corners. We can smooth out the transversal corners by Lemma 2.9 to get the required disk $\widehat{D}$.

\section{Tight CONTACT StruCture ON ClOSED SOlid TORI}

This section will be devoted to the study of tight contact structures on closed solid tori. The results will be primarily of positive nature; that is, we will show that, under certain conditions, two tight contact structures are in the same diffeomorphism class (i.e. they are contactomorphic). The basic tool which will be used in the proofs of most of the results below will be the following proposition which is a more or less direct application of Proposition 2.7 and Lemma 2.10.

Proposition 5.1. Let $M_{1}$ and $M_{2}$ be two closed solid tori with tight contact structure. Then $M_{1}$ and $M_{2}$ are contactomorphic iff the following conditions are satisfied.

(1) $\partial M_{1}$ and $\partial M_{2}$ are contactly equivalent via a c-extendable map $f$;

(2) there exist $D_{i} \subset M_{i}$, disks transversal to $\partial M_{i}$, which are contactly equivalent via a c-extendable map $g$;

(3) $\partial D_{i} \subset \partial M_{i}$;

(4) $\partial D \pitchfork \xi$ (the boundary is a transversal curve);

(5) $f_{\mid \partial D_{1}}=g$.

If the conditions above are satisfied, then $f$ can be extended to a contactomorphism.

Proof. We need only apply Proposition 2.7 and Lemma 2.10 to the balls obtained by cutting the solid tori along the disks $D_{i}$.

The above theorem is not very helpful by itself. While the condition (1) is a reasonable one, the requirements (2)-(4) are not really very natural, nor are they easy to check. The next few theorems will be devoted to giving conditions sufficient for the existence of disks and the c-extendable map $g$ which satisfy conditions (2)(4).

Theorem 5.2. Let $M_{1}$ and $M_{2}$ be two closed solid tori with tight contact structure and normal boundaries. Also assume that

(1) $1\left(M_{1}\right)=1\left(M_{2}\right)$, and

(2) $\operatorname{conj}_{\Phi}\left(M_{1}\right)=\operatorname{conj}_{\Phi}\left(M_{2}\right)=\{\operatorname{Id}\}$, where Id is the identity diffeomorphism of the circle.

Then $M_{1}$ and $M_{2}$ are contactomorphic. Furthermore, if a c-extendable diffeomorphism of the boundaries maps meridians to meridians then it can be extended to a contactomorphism of the two tori.

Note that condition (2) means that the Poincaré return map of any essential curve is the identity diffeomorphism.

Proof. Let $f$ be such a c-extendable diffeomorphism of the boundaries. Let $\Gamma \subset$ $\partial M_{1}$ be a transversal meridian. The curve $f(\Gamma)$ is also a transversal meridian, so we have $l(\Gamma)=l(f(\Gamma))=l\left(M_{i}\right)=-n$. We can now choose a disk $D_{1} \subset M_{1}$ which spans $\Gamma$ and intersects $\partial M_{1}$ transversely. Lemma 4.6 implies that there exists a disk $D_{2} \subset M_{2}$ which spans $f(\Gamma)$, intersects $\partial M_{2}$ transversely, and has a characteristic foliation which is diffeomorphic to the characteristic foliation of $D_{1}$. Therefore, $D_{i}$ are diffeomorphic via a c-extendable diffeomorphism $g$. This diffeomorphism does not have to agree with $f$ on $\Gamma_{1}$, however. 
Let $\eta=g^{-1} \circ f_{\mid \Gamma_{1}}$. The function $\eta$ maps $\Gamma_{1}$ diffeomorphically onto itself; therefore, it can be induced as a time one map of a time dependent vector field $\nu_{t}^{\prime}$, tangent to $\Gamma_{1}$. Since $\operatorname{conj}_{\phi}\left(M_{1}\right)=\{\operatorname{Id}\}$, the characteristic foliations of $\partial M_{1}$ consist of closed leaves which intersect any essential curve only once. Thus it is possible to extend $\nu_{t}^{\prime}$ to a vector field $\nu_{t}$ on $\partial M_{1}$ in such a way that the induced diffeomorphism of the boundary preserves the characteristic foliation. This, in turn, implies that the diffeomorphism can be extended to a contactomorphism of $M_{1}$ to itself. Call this contactomorphism $h$. By construction $h_{\mid \Gamma_{1}}=\eta$. The c-extendable map between $h^{-1}\left(D_{1}\right)$ and $D_{2}$ is given by $g \circ h$. Now $g \circ h_{\mid \Gamma_{1}}=g \circ \eta=g \circ g^{-1} \circ f_{\mid \Gamma_{1}}=f_{\mid \Gamma_{1}}$, so it satisfies the conditions given in Proposition 5.1 above; thus the two solid tori are contactomorphic via a contactomorphism which extends $f$.

In the next lemma, we shall exhibit a model for a solid torus with any given (normal) boundary, and the essential curve of self-linking number -1 . This lemma is a direct application of Lemma 4.4.

Lemma - Construction 5.3. For any $\phi: \mathbb{S}^{1} \rightarrow \mathbb{S}^{1}=\mathbb{R} / 2 \pi \mathbb{Z}$ a diffeomorphism of a circle which preserves orientation, there exists $M(1, \phi)$, a solid torus with tight contact structure, such that $\mathrm{l}(M(1, \phi))=-1$ and $\phi \in \operatorname{conj}_{\Phi}(M(1, \phi))$.

Proof. By Lemma 4.4 there exists a function $f: T^{2}=\mathbb{R}^{2} /(2 \pi \mathbb{Z} \oplus \mathbb{Z})=(\theta, z) \rightarrow \mathbb{R}^{>0}$ such that the boundary of the solid torus $K_{f}=\{r \leq f(\theta, z)\} \subset \mathbb{T}$ has the necessary characteristic foliation, so that $\phi \in \operatorname{conj}_{\Phi}\left(K_{f}\right)$.

At the same time $\mathrm{l}\left(K_{f}\right)=-1$ since the curve $\{r=f(\theta, 0) ; z=0\} \subset \partial K_{f}$ is the boundary of the disk $\{r \leq f(\theta, 0) ; z=0\} \subset K$ and the characteristic foliation of that disk has only one elliptic singularity at $r=0$. Thus $M(1, \phi)=K_{f}$.

Theorem 5.2 shows that when the behavior of the contact structure near the boundary is extremely simple, then the self-linking number of the essential curve basically determines the contactomorphism type of the structure (if the contact structure is tight). The next few theorems will be devoted to proving a "complementary" statement which says that if an essential curve is particularly simple (which means it has a self-linking number equal to -1), then the behavior on the boundary is the only other invariant of a tight contact structure.

Theorem 5.4. Let $M_{1}, M_{2}$ be solid tori with tight contact structures and normal boundaries. Assume $\mathrm{l}\left(M_{1}\right)=\mathrm{l}\left(M_{2}\right)=-1$; then the two tori are contactomorphic iff $\operatorname{conj}_{\Phi}\left(M_{1}\right)=\operatorname{conj}_{\Phi}\left(M_{2}\right)$. Furthermore, if a c-extendable diffeomorphism of the boundaries maps meridians to meridians then it can be extended to a contactomorphism of the two tori.

Before proving this theorem we shall have to prove an auxiliary result which will be necessary in the proof. This is a technical result which should be thought of as a generalization of Theorem 5.2.

Auxiliary Lemma 5.5. Let $M_{1}$ and $M_{2}$ be two closed solid tori with tight contact structure and normal boundaries. Assume $\mathrm{l}\left(M_{1}\right)=\mathrm{l}\left(M_{2}\right)=-n$ and $\operatorname{conj}_{\phi}\left(M_{1}\right)=$ $\operatorname{conj}_{\Phi}\left(M_{2}\right)$. Also assume that there exist a solid torus $N \in M_{1}$ and an annulus $A \subset \overline{M_{1} \backslash N}$ such that:

(1) $\partial N$ is normal;

(2) $\operatorname{conj}_{\Phi}(N)=\{\mathrm{Id}\}$

(3) $\overline{M_{1} \backslash N}$ is diffeomorphic to $\partial M_{1} \times[0,1]$; 
(4) the characteristic foliation of $A$ has no singularities;

(5) $A \pitchfork \partial M$ and $A \pitchfork \partial N$;

(6) $A \cap \partial M=\Gamma$ and $A \cap \partial N=\Gamma^{\prime}$ where $\Gamma$ and $\Gamma^{\prime}$ are both transversal.

Then $M_{1}$ and $M_{2}$ are contactomorphic. Furthermore, if a c-extendable diffeomorphism of the boundaries maps meridians to meridians then it can be extended to a contactomorphism of the two tori.

Proof. Let $f$ be such a c-extendable diffeomorphism of the boundaries. Choose a disk $D_{1} \subset M_{1}$ which spans $\Gamma$ and contains the annulus $A$. Just as in the proof of Theorem 5.2, we can choose a disk $D_{2} \subset M_{2}$ which spans $f(\Gamma)$ and is diffeomorphic to $D_{1}$ via a c-extendable diffeomorphism $g$.

We now proceed with the construction similar to the one done at the end of the proof of Theorem 5.2 in order to get a diffeomorphism which coincides with $f$ on $\Gamma$.

We can choose the coordinates on $A=\mathbb{S}^{1} \times[0,1]$ in such a way that $\Gamma_{1}=\mathbb{S}^{1} \times\{0\}$ and $\Gamma^{\prime}=\mathbb{S}^{1} \times\{1\}$ and the curves $\{p\} \times[0,1] \subset A$ are Legendrian for any $p \in \mathbb{S}^{1}$ (i.e. these curves give the characteristic foliation of $A$ ).

Define also another annulus $B=\mathbb{S}^{1} \times[1,1+2 \varepsilon] \subset D_{1} \cap N$ where $\mathbb{S}^{1} \times\{1\}=\Gamma^{\prime}$; and $p \times[1,1+2 \varepsilon]$ is Legendrian for any $p \in \mathbb{S}^{1}$. Such an annulus exists since some neighborhood of $\Gamma^{\prime}$ in $D_{1}$ must contain no singularities. Let $B^{\prime}=\mathbb{S}^{1} \times[1,1+\varepsilon] \subset B$. Finally, define $A^{\prime}=A \cup B^{\prime}=\mathbb{S}^{1} \times[0,1+\varepsilon]$.

Let $\eta=g^{-1} \circ f_{\mid \Gamma_{1}}$. It is a diffeomorphism of $\mathbb{S}^{1}=\Gamma_{1}$ onto itself; therefore, it can be induced by a time dependent vector field $\nu_{t}^{\prime \prime}$, tangent to $\Gamma_{1}$. This vector field can be extended to a vector field $\nu_{t}^{\prime}$ on $A^{\prime}$ in such a way that $\nu_{t}^{\prime}$ is tangent to $\Gamma^{\prime}$ and $\nu_{t}^{\prime}$ preserves the characteristic foliation.

Let $\hat{h}$ be the diffeomorphism that is induced by $\nu_{t}^{\prime}$.

The vector field $\nu_{t \mid \Gamma^{\prime}}^{\prime}$ can be extended, once again, to a vector field on $\partial N$. This vector field can, in turn, be extended to $\nu_{t}$, a contact vector field on $N$. Note, however, that $\nu_{t}$ can be chosen to coincide with $\nu_{t}^{\prime \prime}$ on $B^{\prime}$. This contact vector field on $N$ integrates to a contactomorphism $h$ which takes $N$ to itself and has the following properties:

(1) $h\left(B^{\prime}\right)=B^{\prime}$

(2) $h_{\mid B^{\prime}}=\hat{h}_{\mid B^{\prime}}$.

Property (1) implies that the disk $A \cup h^{-1}\left(D_{1} \cap N\right)$ is actually a smooth disk. Property (2) implies that the map from $A \cup h^{-1}\left(D_{1} \cap N\right)$ to $D_{1}$ defined by $\hat{h} \cup h$ is a well defined smooth map which is c-extendable. The disk $A \cup h^{-1}\left(D_{1} \cap N\right)$ and the map $g \circ(\hat{h} \cup \tilde{h})$ satisfy the necessary properties given in Proposition 5.1, so $f$ can be extended to a contactomorphism.

We now proceed with the proof of Theorem 5.4.

Proof of 5.4. Instead of showing that $M_{1}$ is contactomorphic to $M_{2}$, we shall show that for any $\phi \in \operatorname{conj}_{\Phi}\left(M_{i}\right), M_{i}$ are contactomorphic to $M(1, \phi)$.

To do that we shall show that $M(1, \phi)$ satisfies the conditions of the Lemma 5.5.

In other words, we shall construct a solid torus $N \subset M(1, \phi)$ which satisfies the necessary properties. Note that since $f: T^{2} \rightarrow \mathbb{R}^{>0}, \min f>0$.

Let $k \in \mathbb{Z}^{>0}$ such that $(2 k \pi)^{-1 / 2}<\min f$. 
Let $N=\left\{r \leq(2 k \pi)^{-1 / 2}\right\}$. A simple calculation shows that the curves $\gamma_{\alpha}(t)=$ $\{z=t, \theta=(2 k \pi) t+\alpha, r=f(z, \theta)\}$ for $t \in[0,1]$ give the characteristic foliation of $\partial N$. Thus

(1) $N \subset M(1, \phi)$;

(2) $\partial N$ is normal;

(3) $\operatorname{conj}_{\Phi}(N)=\{\operatorname{Id}\}$;

(4) $\overline{M(1, \phi) \backslash N}$ is diffeomorphic to $\partial M(1, \phi) \times[0,1]$.

The annulus given by $A=\left\{z=0,(2 k \pi)^{-1 / 2} \leq r \leq f(0, \theta)\right\} \subset \overline{M(1, \phi) \backslash N}$ satisfies the properties (5)-(7) of Lemma 5.5; therefore, we are done.

Definition 5.6. A solid torus $M, \xi$ will be called simple if

(1) $\xi$ is tight;

(2) $\partial M$ is normal;

(3) $\mathrm{l}(M)=-1$.

\section{OPEN SOLID TORI I}

This section shall be devoted to the study of the invariants of tight contact structures on the interior of a closed solid torus. We shall prove that $\mathrm{r}(K)$ and $\mathrm{l}(K)$ are actually invariants of the interior. This fact is essentially a statement about the structure of the tight contact structure in the neighborhood of the boundary of a closed solid torus. The proof requires, however, the following "global" result.

Monotonicity Theorem (Theorem 8.1). Let $K, K^{\prime}$ be two simple solid tori. If there exists a contactomorphism $\phi: K^{\prime} \rightarrow \stackrel{\circ}{K}$ such that $\overline{K \backslash \phi\left(K^{\prime}\right)} \cong \partial K \times[0,1]$ then $\mathrm{r}(K) \leq \mathrm{r}\left(K^{\prime}\right)$.

The proof of this theorem is complicated enough that it merits its own section; it shall be taken for granted here. It is important to note that the statement $\mathrm{r}(K) \leq \mathrm{r}\left(K^{\prime}\right)$ should be interpreted with care. As we explained in section 4 , it is normally impossible to compare the rotation numbers of the characteristic foliation of boundaries of two different solid tori, since the rotation numbers are only defined $\bmod 2 \pi$. In this case, however, the comparison of the rotation numbers becomes meaningful because the contactomorphism $\phi$ induces an isomorphism in homology of the two solid tori (see section 4).

This section shall be devoted to the proof of the following theorem:

Theorem 6.1. If $K, K^{\prime}$ are two solid tori with normal boundaries such that there exists a contactomorphism $\phi: \stackrel{\circ}{K} \rightarrow \stackrel{\circ}{K^{\prime}}$ then $\mathrm{r}(K)=\mathrm{r}\left(K^{\prime}\right)$ and $\mathrm{l}(K)=\mathrm{l}\left(K^{\prime}\right)$.

The proof will basically show that the behavior of the characteristic foliation of the boundary can be "approximated" from the inside. This requires a few local monotonicity results about the properties of the rotation and the self-linking invariants. These are done in Lemmas 6.3 and 6.5 below. The proof of 6.1 will follow afterwards.

To simplify the notation in the next few sections, we shall introduce a partial order on solid tori:

Definition 6.2. Let $K$ and $K^{\prime}$ be two solid tori each equipped with a contact structure. When there exists a contactomorphism $\phi: K^{\prime} \rightarrow \stackrel{\circ}{K}$ such that $\overline{K \backslash \phi\left(K^{\prime}\right)}$ is homeomorphic to $\partial K \times[0,1]$, we shall write that $K^{\prime} \stackrel{\phi}{\prec} K$. 
Lemma 6.3 (Local Monotonicity). Let $K$ be a solid torus with a normal boundary, $\partial K=T$. There exists a tubular neighborhood $U$ of $T$ such that for any $K^{\prime} \stackrel{\gtrless}{\prec} K$ if $\phi\left(\partial K^{\prime}\right)=\partial \phi\left(K^{\prime}\right) \subset U$ and $\partial K$ is normal then $\mathrm{l}\left(K^{\prime}\right) \leq \mathrm{l}(K)$; and if $\mathrm{l}\left(K^{\prime}\right)=\mathrm{l}(K)$ then $\mathrm{r}\left(K^{\prime}\right) \geq \mathrm{r}(K)$.

Proof. Lemma 4.4 implies that there exists a normal torus $N$ such that $\partial N$ is contactly equivalent (in the sense of Definition 2.1) to $T$ and $\mathrm{l}(N)=-1$. Therefore, there exists a neighborhood $U$ of $T$ and a contactomorphism $\psi: U \rightarrow N$ such that $\psi(T)=\partial N$. If $K^{\prime} \stackrel{\phi}{\prec} K$, and $\phi\left(\partial K^{\prime}\right) \subset U$ then $\psi \circ \phi\left(\partial K^{\prime}\right)$ is a boundary of some solid torus $N^{\prime} \subset N$. Now since $\psi$ is a contactomorphism the following equations hold:

(1) $\mathrm{r}(K)=\mathrm{r}(N)$

(2) $\mathrm{r}\left(K^{\prime}\right)=\mathrm{r}\left(N^{\prime}\right)$;

(3) $1(K)-1\left(K^{\prime}\right)=1(N)-1\left(N^{\prime}\right)$.

At the same time the Bennequin inequality implies that $\mathrm{l}(N)-\mathrm{l}\left(N^{\prime}\right) \geq 0$ so $\mathrm{l}\left(K^{\prime}\right) \leq$ $\mathrm{l}(K)$. In the case when $\mathrm{l}\left(K^{\prime}\right)=\mathrm{l}(K)$ Theorem 8.1 implies that $\mathrm{r}\left(N^{\prime}\right) \geq \mathrm{r}(N)$.

Definition 6.4. If $K$ is a solid torus with normal boundary then any neighborhood of $\partial K$ which has the properties described in the Lemma 6.3 will be called $a$ neighborhood of monotonicity.

Lemma 6.5. Let $K$ be a solid torus with a normal boundary $T=\partial K$. There exists a family of solid tori $\left\{K_{n}\right\} \subset K$ such that

(1) $K^{\prime} \stackrel{\phi}{\prec} K$, where $\phi$ is the inclusion contactomorphism;

(2) $K_{n} \subset K_{n+1}$;

(3) $\cup_{n} K_{n}=\stackrel{\circ}{K}$;

(4) $\mathrm{l}\left(K_{n}\right)=\mathrm{l}(K)$;

(5) $\mathrm{r}\left(K_{n}\right) \geq \mathrm{r}\left(K_{n+1}\right)$;

(6) $\mathrm{r}\left(K_{n}\right) \geq \mathrm{r}(K)$;

(7) $\lim _{n \rightarrow \infty} \mathrm{r}\left(K_{n}\right)=\mathrm{r}(K)$.

Proof. As in Lemma 6.3 above there exist

(1) a normal torus $N$ such that $\mathrm{l}(N)=-1$;

(2) a neighborhood $U$ of $T$;

(3) a contactomorphism $\phi: U \rightarrow N$ such that $\phi(T)=\partial N$.

By Lemma 4.4 the solid torus $N$ can be chosen to be a set of the form $N=$ $\{r \leq f(z, \theta)\} \subset \mathbb{T}$ where $\mathbb{T}=\mathbb{R}^{3} /\{z \mapsto z+1\}$ with the contact structure given by the kernel of $d z+r^{2} d \theta$, and $f$ is a twice periodic, smooth, positive function, $f: \mathbb{R}^{2} /(\mathbb{Z} \oplus 2 \pi \mathbb{Z}) \rightarrow \mathbb{R}^{>0}$. Consider the solid tori $N_{n}=\{r \leq(1-1 / n) \cdot f(z, \theta)\} \subset \mathbb{T}$. By Lemma 4.5 these have normal boundaries. Furthermore

(1) $\mathrm{l}\left(N_{n}\right)=-1=\mathrm{l}(N)$;

(2) $\mathrm{r}\left(N_{n}\right) \geq \mathrm{r}\left(N_{n+1}\right) \geq \mathrm{r}(N)$ (by the monotonicity theorem, see section 8);

(3) $\lim _{n \rightarrow \infty} \mathrm{r}\left(N_{n}\right)=\mathrm{r}(N)$.

Now each torus $\phi^{-1}\left(\partial N_{n}\right)$ is a boundary of some solid torus $K_{n} \in K$. The family $\left\{K_{n}\right\}$ is the required family of tori.

Now we can proceed with the proof of Theorem 6.1. 
Proof of Theorem 6.1. Suppose that $\psi: \stackrel{\circ}{K} \rightarrow \stackrel{\circ}{K}$ is a contactomorphism. Let $\left\{K_{n}\right\} \subset K$ be the family of solid tori which satisfy the properties (1)-(7) of Lemma 6.5 and let $\left\{K_{n}^{\prime}\right\} \subset K^{\prime}$ also be such a family. Note that $\bigcup_{n} \psi\left(K_{n}\right)=\stackrel{\circ}{K^{\prime}}$ and $K_{n} \stackrel{\psi}{\prec} K^{\prime}$. Also $\bigcup_{n} \psi^{-1}\left(K_{n}^{\prime}\right)=\stackrel{\circ}{K}$, and $K_{n}^{\prime} \stackrel{\psi^{-1}}{\prec} K$. Let $U$ be a neighborhood of monotonicity of $\partial K$ and let $U^{\prime}$ be a neighborhood of monotonicity of $\partial K^{\prime}$ (these neighborhoods exist by Lemma 6.3). There exists a number $N_{0} \in \mathbb{Z}^{>0}$ such that if $n \geq N_{0}$, then $\psi\left(\partial K_{n}\right) \subset U^{\prime}$; therefore we have $\mathrm{l}\left(K_{n}\right)=\mathrm{l}(K) \leq \mathrm{l}\left(K^{\prime}\right)$. Equivalently, we must have $\mathrm{l}\left(K_{n}^{\prime}\right)=\mathrm{l}\left(K^{\prime}\right) \leq \mathrm{l}(K)$, so $\mathrm{l}(K)=\mathrm{l}\left(K^{\prime}\right)$ because the argument is symmetric.

Now assume (without loss of generality) that $\mathrm{r}\left(K^{\prime}\right) \geq \mathrm{r}(K)+\delta$ for some $\delta>0$. We know (property (4) Lemma 6.5) that $\mathrm{l}\left(K_{n}\right)=\mathrm{l}(K)$, and we just proved that $\mathrm{l}(K)=\mathrm{l}\left(K^{\prime}\right)$ so $\mathrm{l}\left(\psi\left(K_{n}\right)\right)=\mathrm{l}\left(K^{\prime}\right)$. Thus Lemma 6.3 implies that if $\psi\left(\partial K_{n}\right) \subset U^{\prime}$ then $\mathrm{r}\left(K_{n}\right) \geq \mathrm{r}\left(K^{\prime}\right)$. At the same time, we know that there exists a positive number $N_{1} \in \mathbb{Z}^{>0}$ such that if $n \geq N_{1}$, then $\mathrm{r}\left(K_{n}\right)<\mathrm{r}(K)+\frac{\delta}{2}$. Therefore, for $n \geq \max \left(N_{0}, N_{1}\right)$ we would have $\mathrm{r}\left(K^{\prime}\right)>\mathrm{r}\left(\psi\left(K_{n}\right)\right)$, but this cannot happen so $\mathrm{r}\left(K^{\prime}\right) \leq \mathrm{r}(K)$. This argument is also symmetric with respect to $K$ and $K^{\prime}$ which means that $\mathrm{r}(K)$ must be equal to $\mathrm{r}\left(K^{\prime}\right)$.

The results of this section enable us to define a self-linking number for any tight torus.

Definition 6.6. Let $K$ be a solid torus with a tight contact structure. Define $\left.\left.\mathrm{l}(K)=\max _{\left\{K_{n}^{\prime}\right\}}\right\} \lim _{n \rightarrow \infty} \mathrm{l}\left(K_{n}^{\prime}\right)\right]$ where $\left\{K_{n}^{\prime}\right\}^{\infty} \subset K$ is a family of nested normal solid tori such that $K_{n}^{\prime} \prec K$ and $\bigcup_{n=1}^{\infty} K_{n}^{\prime}=K$.

In the case when $\mathrm{l}(K)>-\infty$ we can also define the rotation number similarly.

Note that the results of this section imply that this definition coincides with the definitions of the rotation number and the self-linking number for the case of normal boundary.

\section{OPEN SOLID TORI II}

This section is devoted to the study of the invariants defined in the previous section. We shall show that, in general, the $\mathrm{l}(T)$ and $\mathrm{r}(T)$ do not determine contactomorphism class of contact structure.

Lemma 7.1. Let $K$ be a closed solid torus with tight contact structure.

Assume that:

(1) $\partial K$ is convex with respect to some contact vector field $\nu$, and

(2) $1(K)=-1$.

Then there exists another closed solid torus $L$ with tight contact structure such that $\mathrm{l}(K)=\mathrm{l}(L)$ and $\mathrm{r}(K)=\mathrm{r}(L)$, but the interior of $L$ is not contactomorphic to the interior of $K$.

We remind the reader that a convex surface is a surface which is transversal to some contact vector field (see 1.2).

Proof. Let $q=\mathrm{r}(K)$, and consider the solid torus $L=\{r \leq \rho\} \subset \mathbb{T}$ where $\rho=q^{-2}$. It is not difficult to see that $\mathrm{r}(L)=q$ and $\mathrm{l}(L)=-1$.

We shall now proceed to show that $\stackrel{\circ}{L} \not \approx \stackrel{\circ}{K}$. Consider a solid torus $L_{\varepsilon}=$ $\{r \leq \rho \cdot(1-\varepsilon)\} \subset L$, and note that: 
(1) $L_{\varepsilon} \prec L$;

(2) $\mathrm{l}\left(L_{\varepsilon}\right)=-1$;

(3) for any neighborhood of $\partial L$ there exists an $\varepsilon$ small enough that $\partial L_{\varepsilon}$ is in that neighborhood;

(4) $\mathrm{r}\left(L_{\varepsilon}\right)<q$.

On the other hand, there exists a neighborhood $U$ of $\partial K$ such that if a solid torus $K^{\prime} \subset K$ satisfies the following conditions:

(1) $K^{\prime} \prec K$;

(2) $\mathrm{l}\left(K^{\prime}\right)=-1$

(3) $\partial K \subset U$,

then $\mathrm{r}\left(K^{\prime}\right)=q$.

Indeed, choose a neighborhood of monotonicity $V$ of $K$ (which exists by Lemma 6.3). Now consider a contactomorphism $\phi_{\delta}$ induced by a time one map of the contact vector field $\delta \cdot \nu$ (we assume that $\nu$ points inward). If $\delta$ is chosen small enough then

(1) $\phi_{\delta}(\partial K)=\partial\left(\phi_{\delta}(K)\right) \subset V$;

(2) $\phi_{\delta}(K) \varsubsetneqq K$;

(3) $K \stackrel{\phi_{\delta}}{\prec} K$.

Let $U=K \backslash \phi_{\delta}(K)$. We have $U \subset V$; therefore, it is also a neighborhood of monotonicity of $\partial K$. Furthermore $\mathrm{r}\left(\phi_{\delta}(K)\right)=q$. So $U$ is the neighborhood described above.

Now if $\psi: L \rightarrow K$ is a contactomorphism, then the construction above (together with the definition of neighborhood of monotonicity) implies that $\psi\left(L_{\varepsilon}\right) \subset \phi_{\delta}(K)$. This cannot happen so $\stackrel{\circ}{L}$ and $\stackrel{\circ}{K}$ are not contactomorphic.

\section{The Monotonicity THEOREM}

In this section we shall present the proof of the Monotonicity Theorem which was used in section 6 . We begin by recalling the definition of a partial order relation on solid tori from that section.

Definition 6.2. Let $K$ and $K^{\prime}$ be two solid tori each equipped with a contact structure. When there exists a contactomorphism $\phi: K^{\prime} \rightarrow \stackrel{\circ}{K}$ such that $\overline{K \backslash \phi\left(K^{\prime}\right)}$ is homeomorphic to $\partial K \times[0,1]$, we shall write that $K^{\prime} \stackrel{\phi}{\prec} K$.

As we mentioned earlier, $\psi$ induces an isomorphism between the homology of $\partial K$ and the homology of $\partial K^{\prime}$ and this isomorphism takes the homology class of the meridian to the class of the meridian. This allows us to compare the rotation numbers of the boundaries (see section 4).

Monotonicity Theorem (Theorem 8.1). Let $K, K^{\prime}$ be simple solid tori. If $\mathrm{l}(K)=\mathrm{l}\left(K^{\prime}\right)=-1$ and if $K^{\prime} \stackrel{\phi}{\prec} K$ then $\mathrm{r}(K) \leq \mathrm{r}\left(K^{\prime}\right)$.

Before stating the proof we remind the reader that $\mathbb{T}$ represents $\mathbb{R}^{3} /\{z \mapsto z+1\}$ with the contact structure given by the kernel of $d z+r^{2} d \theta$.

Proof. We will basically show that if this inequality were violated, then $\mathbb{T}$ would have to be overtwisted, which is not the case. 
For simplicity we shall identify $K^{\prime}$ with $\phi\left(K^{\prime}\right)$; this will mean that $K^{\prime} \subset K$ and $K^{\prime} \prec K$.

Let $T=\partial K ; T^{\prime}=\partial K^{\prime}$.

Let $\Gamma \subset T, \Gamma^{\prime} \subset T^{\prime}$ be essential curves.

And, finally let $r=\mathrm{r}(K), r^{\prime}=\mathrm{r}\left(K^{\prime}\right)$; we can assume that the generators in $H_{1}(T)$ are chosen in such a way that $r, r^{\prime}>0$.

The proof will be split into several parts. These will be stated as lemmas.

Part 1 (Lemma 8.2). There exists an annulus $A \subset K$ such that

(1) $A \subset \overline{K \backslash K^{\prime}}$;

(2) $\partial A=\Gamma^{\prime} \cup \Gamma$

(3) $A \pitchfork \partial K$ and $A \pitchfork \partial K^{\prime}$;

(4) $A \pitchfork \xi$ (i.e. the characteristic foliation of $A$ has no singularities).

This is a direct consequence of Lemma 3.10 .

Proof. Let $A^{\prime}$ be any annulus satisfying (1) through (3) (these are generic conditions). The curve $\Gamma^{\prime}$ spans a disk $D$ in $T^{\prime}$ which has only one elliptic singularity. Therefore, the annulus $A^{\prime}$ satisfies all the criteria of Lemma 3.10, so all the singularities of the characteristic foliation of $A^{\prime}$ can be killed via a $\mathcal{C}^{0}$ small deformation fixed near the boundary. The resulting annulus $A$ will satisfy (4) as well as (1) through (3).

Part 2 (Lemma 8.3). There exists a family of piecewise smooth tori $T_{\delta}^{\prime}$ which satisfy the following conditions:

(1) $T_{\delta}^{\prime} \subset \overline{K \backslash K^{\prime}}$

(2) the singularities of $T_{\delta}^{\prime}$ are of transversal corner type with respect to $\xi$;

(3) $\Gamma^{\prime} \subset T_{\delta}^{\prime}$ (where $\Gamma^{\prime}$ is an essential curve of $\left.\partial K^{\prime}\right)$;

(4) for any $\varepsilon>0$ there exists a $\delta(\varepsilon)>0$ such that $\left|\mathrm{r}_{\left[\Gamma^{\prime}\right]}\left(T_{\delta}^{\prime}\right)-\mathrm{r}(K)\right|<\varepsilon$ for any $\delta<\delta(\varepsilon)$

The proof of this lemma is a relatively straightforward construction. The constructed tori will consist of four annuli: $D_{\delta}^{c}, A_{\delta}, D_{\delta}^{\prime}$, and $A$.

$D_{\delta}^{c}$ will be a complement of a neighborhood of $\Gamma$ in $T, D_{\delta}^{\prime}$ will be the closure of a small neighborhood of $\Gamma^{\prime}$ in $T^{\prime}$, and $A_{\delta}$ will be a push-off of the given annulus $A$. Probably this construction can be best understood in terms of a picture shown in Figure 3; nevertheless below is a rigorous description.

Proof. Let $B$ be a neighborhood of $\Gamma$ in $T$ which has a transversal boundary. Also let $B^{\prime}$ be a neighborhood of $\Gamma^{\prime}$ in $T^{\prime}$ with the same properties. Both $B$ and $B^{\prime}$ are annuli with transversal boundaries which are transversal to the contact structure. It is easy to verify that $\left\{A, B, B^{\prime}\right\}$ make a triple of smooth surfaces with transversal corners with respect to $\xi\left((1) B \cap A=\Gamma\right.$ and $B^{\prime} \cap A=\Gamma^{\prime} ;(2) A \pitchfork B, B^{\prime}$ since $A \pitchfork T, T^{\prime}$; (3) $B \cap B^{\prime}=\varnothing$ so $B \pitchfork B^{\prime}$; (4) all the boundaries are transversal by definition). Now let

$$
\begin{aligned}
& C_{1}=\{r \in[1,2] ; z=0\} \subset \mathbb{R}^{3}, \\
& C_{2}=\{r=2 ; z \in[-1,1]\} \subset \mathbb{R}^{3}, \\
& C_{3}=\{r=1 ; z \in[-1,1]\} \subset \mathbb{R}^{3} .
\end{aligned}
$$



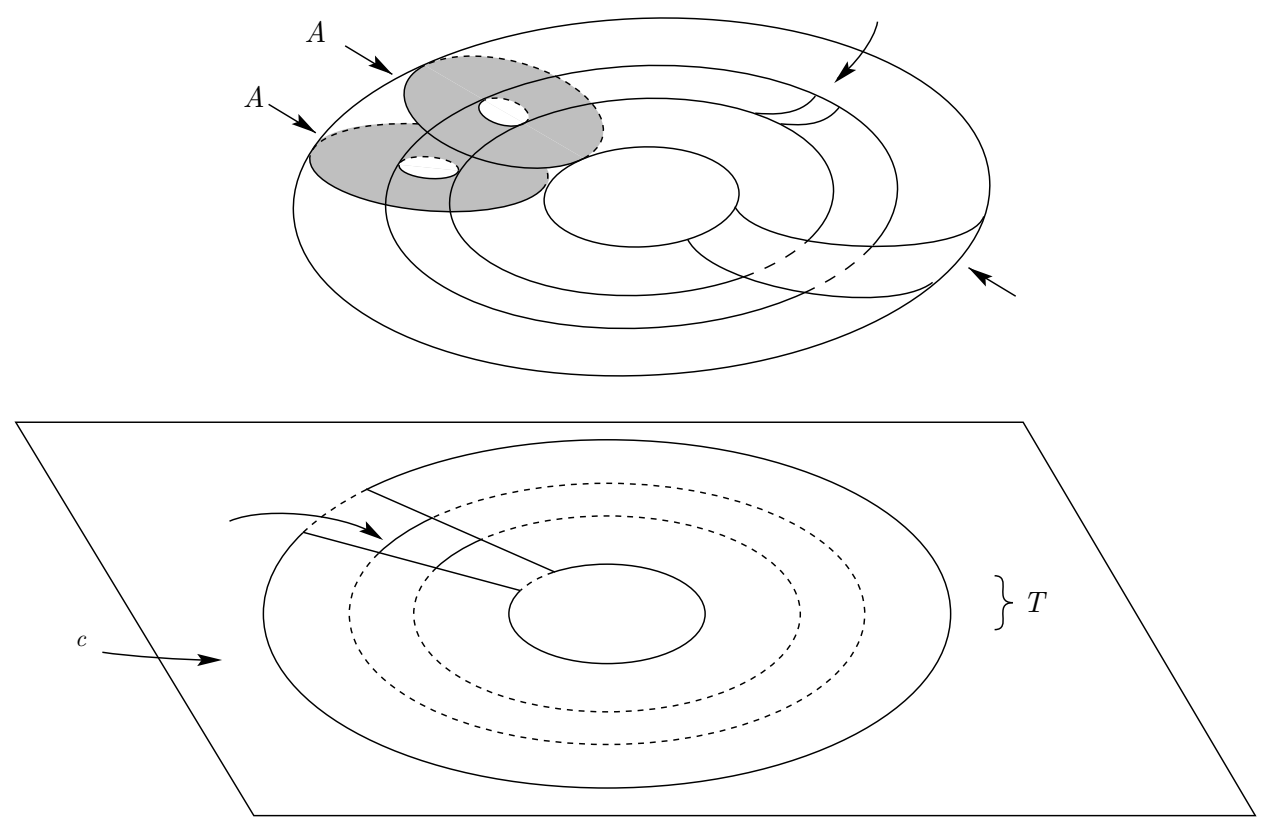

FiguRE $3 . T_{\delta}^{\prime}$

It is easy to see that there exists a family of diffeomorphisms which preserve characteristic foliation and take the family $\left\{A, B, B^{\prime}\right\}$ to $\left\{C_{1}, C_{2}, C_{3}\right\}$. Thus by Proposition 2.7 , the family $\left\{A, B, B^{\prime}\right\}$ is contactly equivalent to the family $\left\{C_{1}, C_{2}, C_{3}\right\}$ which means that there exist a neighborhood $U$ of $A \cup B \cup B^{\prime}$ and a contactomorphism $\phi: U \rightarrow \mathbb{R}^{3}$ such that $\phi\left(\left\{A, B, B^{\prime}\right\}\right)=\left\{C_{1}, C_{2}, C_{3}\right\}$. Now let a contactomorphism $\phi: U \rightarrow \mathbb{R}^{3}$ such that $\phi\left(\left\{A, B, B^{\prime}\right\}\right)=\left\{C_{1}, C_{2}, C_{3}\right\}$. Now let

$$
\begin{aligned}
& C_{1, \delta}=\{r \in[1,2] ; z=\delta\} \subset \mathbb{R}^{3}, \\
& C_{2, \delta}=\{r=2 ; z \in[0, \delta]\} \subset \mathbb{R}^{3}, \\
& C_{3, \delta}=\{r=1 ; z \in[0, \delta]\} \subset \mathbb{R}^{3}, \\
& A_{\delta}=\phi^{-1}\left(C_{1, \delta}\right) \subset K \text {, } \\
& D_{\delta}=\phi^{-1}\left(C_{2, \delta}\right) \subset T \text {, } \\
& D_{\delta}^{c}=\overline{T \backslash \phi^{-1}\left(C_{2, \delta}\right)} \subset T \text {, } \\
& D_{\delta}^{\prime}=\phi^{-1}\left(C_{3, \delta}\right) \subset T^{\prime} \text {, } \\
& \Gamma_{\delta}=A_{\delta} \cap T=\partial D_{\delta} \text {, } \\
& \Gamma_{\delta}^{\prime}=A_{\delta} \cap T=\partial D_{\delta}^{\prime} \text {. }
\end{aligned}
$$

$\delta$ should be chosen small enough that $C_{i, \delta} \subset f(U)$. Consider the torus

$$
T_{\delta}^{\prime}=D_{\delta}^{c} \cup A \cup D_{\delta}^{\prime} \cup A_{\delta} .
$$

It is easy to see that $T_{\delta}^{\prime}$ has transversal corners and that is a normal torus.

By construction $\Gamma^{\prime} \subset T_{\delta}^{\prime}$. 
Since $\Gamma_{\delta} \subset T$ for any $\delta>0$ and $\Gamma_{\delta} \subset T_{\delta}^{\prime}$, it is possible to compare $\Phi_{T, \Gamma_{\delta}}$ with $\Phi_{T_{\delta}^{\prime}, \Gamma_{\delta}}$. A simple calculation shows that any leaf of the characteristic foliation of $C_{2, \delta}$ connects a point $(\theta, 2,0) \in \mathbb{R}^{3}$ with the point $(\theta+\delta / 4,2, \delta) \in \mathbb{R}^{3}$. At the same time the piecewise smooth leaves of $C_{1} \cup C_{3, \delta} \cup C_{1, \delta}$ connect the points $(\theta, 2,0) \in \mathbb{R}^{3}$ with the points $(\theta+\delta, 2, \delta) \in \mathbb{R}^{3}$. Thus, $\Phi_{T, \Gamma_{\delta}}=\Phi_{T_{\delta}^{\prime}, \Gamma_{\delta}} \circ \operatorname{rot}_{(3 / 4) \delta}$ where $\operatorname{rot}_{a}$ represents a rotation by $a$. This equation implies that for any fixed $\delta_{0}$ we have $\lim _{\delta \rightarrow 0} \Phi_{T_{\delta}^{\prime}, \Gamma_{\delta_{0}}}=\Phi_{T, \Gamma_{\delta_{0}}}$. This, in turn, implies that $\lim _{\delta \rightarrow 0} \mathrm{r}_{[\Gamma]}\left(T^{\prime}\right)_{\delta}=\mathrm{r}(K)$, which means that, for any $\varepsilon>0$, there exists a $\delta(\varepsilon)>0$ such that $\left|\mathrm{r}_{[\Gamma]}\left(T_{\delta}^{\prime}\right)-r\right|<\varepsilon$ for any $\delta<\delta(\varepsilon)$.

Part 3 (Lemma 8.4). Let $T^{\prime \prime} \subset K$ be a normal piecewise smooth torus with transversal corners such that:

(1) $T^{\prime \prime} \cap T^{\prime}=D$ where $D$ is a smooth annulus with a transversal boundary;

(2) $\Gamma^{\prime} \subset D \subset T^{\prime \prime}$;

(3) $T^{\prime} \cup T^{\prime \prime} \backslash \stackrel{\circ}{D}$ is also a piecewise smooth torus with transversal corners.

Also assume that $\left|\mathrm{r}_{\left[\Gamma^{\prime}\right]}\left(T^{\prime \prime}\right)-r^{\prime}\right|<2 \pi$. Then the map $\Phi_{T^{\prime \prime}, \Gamma^{\prime}} \circ \Phi_{T^{\prime}, \Gamma^{\prime}}^{-1}$ has no fixed points.

The basic idea behind the proof of this lemma is that if there exists such a point, it is possible to find a disk which has a closed non-singular leaf in its characteristic foliation. This is the crux of the whole proof.

Proof. Let $g$ be the Poincaré return map of $\Gamma^{\prime}$ with respect to $T^{\prime}\left(g=\Phi_{T^{\prime}, \Gamma^{\prime}}\right)$ and $f$ be the Poincaré return map of $\Gamma^{\prime}$ with respect to $T^{\prime \prime}\left(f=\Phi_{T^{\prime \prime}, \Gamma^{\prime}}\right)$. We have to show that the map $f \circ g^{-1}$ has no fixed points. Assume that this is false; then there exists a point $p \in \Gamma^{\prime}$ such that $f(p)=g(p)=p^{\prime} \in \Gamma^{\prime}$. This means that there exist Legendrian imbeddings $\gamma^{\prime}:[0,1] \rightarrow T^{\prime}$ and $\gamma^{\prime \prime}:[0,1] \rightarrow T^{\prime \prime}$ such that $\gamma^{\prime}(0)=\gamma^{\prime \prime}(0)=p$ and $\gamma^{\prime}(1)=\gamma^{\prime \prime}(1)=p^{\prime}$. (These are leaves of the respective characteristic foliations.) Consider the piecewise smooth torus $\widehat{T}=T^{\prime} \cup T^{\prime \prime} \backslash \stackrel{\circ}{D}$. Since $T^{\prime} \cap T^{\prime \prime}=D$, the images of $[0,1]$ under $\gamma^{\prime}$ and $\gamma^{\prime \prime}$ coincide on $D$. Therefore, $\widehat{T}$ will have a closed leaf of the characteristic foliation given by $\operatorname{Im}\left(\gamma^{\prime}\right) \cup \operatorname{Im}\left(\gamma^{\prime \prime}\right) \backslash D$. Now if $\left|\mathrm{r}_{\left[\Gamma^{\prime}\right]}\left(T^{\prime \prime}\right)-r^{\prime}\right|<2 \pi$, this closed cycle is in fact a boundary of a disk. But, by Lemma 2.10 it is possible to smooth out $\widehat{T}$ and to get a smooth torus with characteristic foliation which has a closed leaf which is a meridian. This would imply that $\xi$ is overtwisted, which contradicts our assumption.

We are now ready to prove the Monotonicity Theorem.

\section{Part 4 (Proof of Theorem 8.1).}

Proof. Assume that the inequality is not satisfied, that is, $r>r^{\prime}$.

By Lemma 4.4 there exists a smooth function $f: \mathbb{R}^{2} /(2 \pi \mathbb{Z} \oplus \mathbb{Z})=(\theta, z) \rightarrow \mathbb{R}^{>0}$ such that the set $\{r \leq f(\theta, z)\} \subset \mathbb{T}$ is contactomorphic to $K$. Therefore, we can assume without loss of generality that $K=\{r \leq f(\theta, z)\} \subset \mathbb{T}$. Now consider the set $K_{a}=\{r \leq a \cdot f(z, \theta)\}$. It is easy to see that $K_{a}$ has normal boundary (Lemma 4.5) and the dependence of the vector field which induces the characteristic foliation on $\partial K_{a}$ can be made to depend smoothly on $a$; therefore, the Poincaré return map of $\partial K_{a}$ also depends smoothly on $a$. At the same time $\lim _{a \rightarrow \infty} r\left(\partial \widehat{K}_{a}\right)=0$. Thus, there exists an $a_{0}$ such that $\mathrm{r}\left(K_{a_{0}}\right)>r^{\prime}$ and $\left|\mathrm{r}\left(K_{a_{0}}\right)-r^{\prime}\right|<2 \pi$. Note that $\mathrm{l}\left(K_{a_{0}}\right)=-1$ and $K^{\prime} \prec K_{a_{0}}$. Thus, by replacing $K$ with $K_{a_{0}}$, we can assume 


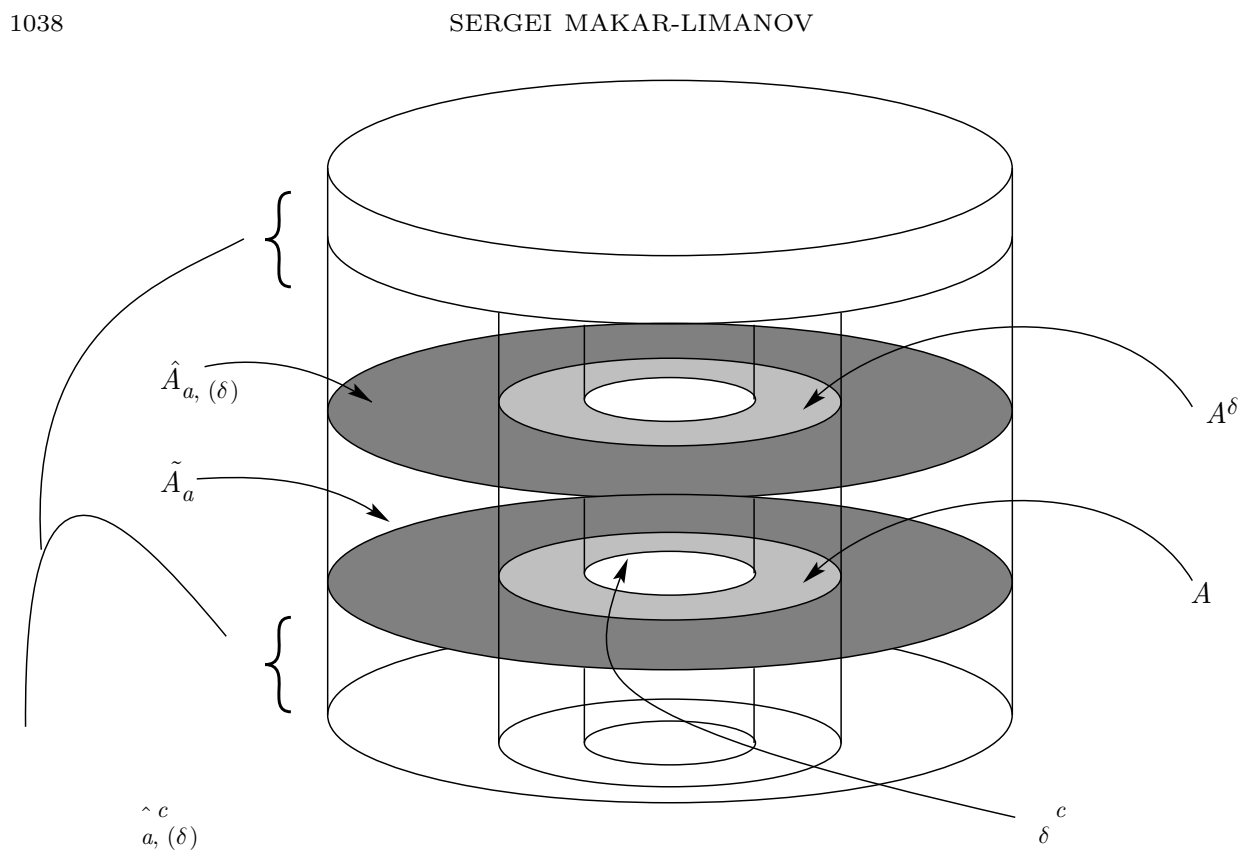

Figure 4. $\widehat{T}_{a}$

without loss of generality that $\left|r-r^{\prime}\right|<2 \pi$. By Lemma 8.3 (Part 2), we can choose a $\delta$ such that

(1) $T_{\delta}^{\prime} \subset \overline{K \backslash K^{\prime}}$;

(2) $r_{\left[\Gamma^{\prime}\right]}\left(T_{\delta}^{\prime}\right)>r^{\prime}$

(3) $r_{\left[\Gamma^{\prime}\right]}\left(T_{\delta}^{\prime}\right)-r^{\prime}<2 \pi$.

We can further choose $\delta$ to be small enough that $\delta<r^{\prime}$. We now construct a whole family of tori $\widehat{T}_{a}$ for $a \in[1, \infty)$. Every torus of this family shall satisfy the conditions of Lemma 8.4 (Part 3), but we shall show that there is at least one torus in that family which violates the conclusion of Lemma 8.4. Once again this construction is best understood in terms of a picture in Figure 4.

The torus $\widehat{T}_{a}$ will consist of a union of six annuli: $\widehat{D}_{a,(\delta)}^{c}, \widetilde{A}_{a}, A, D_{\delta}^{\prime}, A_{\delta}$, and $\widehat{A}_{a,(\delta)}$. Three of those tori, $A, D_{\delta}^{\prime}$, and $A_{\delta}$, were already defined in Part 2 of this proof. The annulus $\widehat{D}_{a,(\delta)}^{c}$ is the annulus $D_{\delta}^{c}$ "stretched" by the parameter $a$, and the annuli $\widetilde{A}_{a}$, and $\widehat{A}_{a,(\delta)}$ will be the annuli which connect the edges of $D_{\delta}^{c}$ and the edges of $A \cup D_{\delta}^{\prime} \cup A_{\delta}$. Below are the rigorous definitions:

$$
\begin{aligned}
\widehat{D}_{a,(\delta)}^{c} & =\left\{(a \cdot r, \theta, z) \mid(r, \theta, z) \in D_{\delta}^{c}\right\} \subset T, \\
\widehat{A}_{a,(\delta)} & =\left\{(r, \theta, z) \mid \exists r^{\prime} \text { such that }\left(r^{\prime}, \theta, z\right) \in \Gamma_{\delta} ; r \in\left[r^{\prime}, a \cdot r^{\prime}\right]\right\} \subset K_{a}, \\
\widetilde{A}_{a} & =\left\{(r, \theta, z) \mid \exists r^{\prime} \text { such that }\left(r^{\prime}, \theta, z\right) \in \Gamma ; r \in\left[r^{\prime}, a \cdot r^{\prime}\right]\right\} \subset K_{a} .
\end{aligned}
$$

We now have

$$
\widehat{T}_{a}=\widehat{D}_{a,(\delta)}^{c} \cup \widetilde{A}_{a} \cup A \cup D_{\delta}^{\prime} \cup A_{\delta} \cup \widehat{A}_{a,(\delta)} .
$$

Let $f_{a}$ be the Poincaré return map of $\Gamma^{\prime}$ with respect to $\widehat{T}_{a}$ (that is, $f_{a}=\Phi_{\widehat{T}_{a}, \Gamma^{\prime}}$ ) and let $g$ be the Poincare return map of $\Gamma^{\prime}$ with respect to $T^{\prime}$ as before. Since 
$\widehat{T}_{1}=T_{\delta}$ we have $r_{\left[\Gamma^{\prime}\right]}\left(\widehat{T}_{1}\right)>r_{\left[\Gamma^{\prime}\right]}\left(T^{\prime}\right)$. This implies that the map $f_{1} \circ g^{-1}$ moves all the points of $\Gamma^{\prime}$ counterclockwise. On the other hand, simple calculation shows that we have $\lim _{a \rightarrow \infty} f_{a}=\operatorname{rot}_{\delta}$. This means that there exists an $a_{0}$ large enough that $f_{a_{0}} \circ g^{-1}$ will move all the points of $\Gamma^{\prime}$ clockwise (this is because $\delta$ was chosen smaller than $r^{\prime}$ ). But the map $f_{a} \circ g^{-1}$ depends continuously on $a$ which means that there must be an $a_{1} \in\left[1, a_{0}\right]$ for which the map $f_{a_{1}} \circ g^{-1}$ has a fixed point. This contradicts Lemma 8.4 implying that the assumption that $r>r^{\prime}$ cannot hold.

\section{Examples of Tight CONTACT STRUCTURES ON SOLID TORI}

We have already constructed some tight contact structures on a solid torus in previous sections. These constructions produced tight contact structures which were in some ways "simple"; in this section we shall construct two examples of tight contact structures on a solid torus which are unusual in some ways.

First we will construct a family of tight contact structures on a solid torus with the property that each contact structure in this family will induce an overtwisted contact structure on a finite cover of the solid torus.

Theorem 9.1. For any $j \in \mathbb{Z}^{>0}$ there exists a solid torus $T_{j} \subset\left(\mathbb{S}^{3}, \xi\right)$ (where $\xi$ is the standard, tight contact structure on $\mathbb{S}^{3}$ ) such that

(1) For some finite-sheeted cover $\psi: \widetilde{T}_{j} \rightarrow T_{j}$ the contact structure $\psi^{*}(\xi)$ is overtwisted;

(2) $\mathrm{l}\left(T_{j}\right)=-2 j-1$.

Proof. Fix $j \in \mathbb{Z}^{>0}$. The proof shall consist of two parts. In the first part we shall construct $T_{j}$. In the second we shall show that the constructed solid torus satisfies the necessary properties.

Part I - Construction: We shall choose a curve $\Gamma \subset \mathbb{S}^{3}$ and a tubular neighborhood $U$ of $\Gamma$ in such a way that the complement of $U$ in $\mathbb{S}^{3}$ will be a solid torus.

Choose a transversal curve $\Gamma \subset \mathbb{S}^{3}$ such that $l(\Gamma)=-2 j-1$ and $\Gamma$ spans a disk (the existence of such curves is well known [El4]).

We now proceed with the construction of $U$ :

Let $K_{a}=\left\{r<(2 a \pi)^{2}\right\} \subset \mathbb{T}$. A simple calculation shows that for $n \in \mathbb{Z}^{>0}$, $\mathrm{l}\left(\overline{K_{\frac{1}{n}}}\right)=-1$, and $\operatorname{conj}_{\phi}\left(\overline{K_{\frac{1}{n}}}\right)=\{\mathrm{Id}\}$. Theorem 5.2 implies that $K_{1}$ is contactomorphic to $K_{\frac{1}{n}}$ (this can also be implied from Theorem 5.4, nor is it too difficult to construct an explicit contactomorphism). Also $\bigcap_{n=1}^{\infty} K_{\frac{1}{n}}=\{r=\theta=0\}$; thus any tubular neighborhood of the transversal curve $\{r=\theta=0\} \subset \mathbb{T}$ contains a tubular neighborhood which is contactomorphic to $K_{1}$. Since any two transversal curves are contactly equivalent (in the sense of Definition 2.1), there is a tubular neighborhood $V$ of $\Gamma$ together with a contactomorphism $\phi: V \rightarrow \mathbb{T}$ such that $\phi(V)=K_{1}$ and $\phi(\Gamma)=\{r=\theta=0\}$. Let $U=\phi^{-1}\left(K_{\frac{1}{2}}\right)$ and $T_{j}=\mathbb{S}^{3} \backslash U$. Figure 5 shows $V, U, \Gamma$, and $D$ for the case when $l(\Gamma)=-3$.

Part II: Since $j>1$ is fixed, let $T=T_{j}$.

Let $\alpha \in H_{1}(\partial T)$ represent the class of the meridian (with respect to $T$ ) and choose $\beta \in H_{1}(\partial T)$ so that $\{\alpha, \beta\}$ spans $H_{1}(\partial T)$. Note that we have an isomorphism $\iota: H_{1}(\partial T) \rightarrow H_{1}(\partial V)$ induced by the inclusion of $\partial V$ into $T$. The characteristic foliation $\partial V$ is a foliation by closed leaves (by construction). If $\gamma$ is the homology 


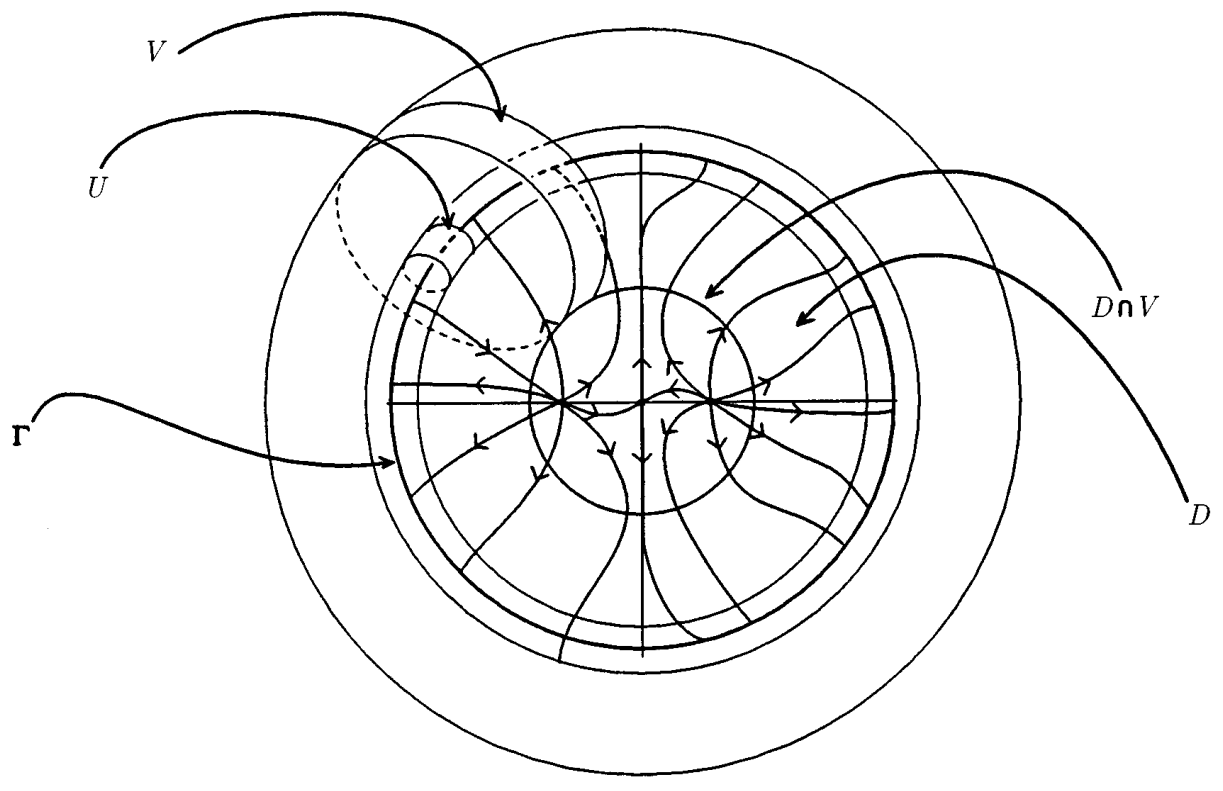

Figure 5. $V, U, \Gamma$, and $D$ if $l(\Gamma)=-3$

class of these leaves we have $\gamma=p \iota(\alpha)+q \iota(\beta)$ for some $p, q \subset \mathbb{Z}$. By choosing the orientation appropriately we can assume that $q>0$.

We shall now show that if $\psi: \widetilde{T} \rightarrow T$ is a $q+1$-sheeted cover of $T$, then $\psi^{*}(\xi)$ is overtwisted (see Figure 6).

We will do this by exhibiting a 2-sphere $S \subset \widetilde{T}$ such that $S_{\psi^{*}(\xi)}$ has a nonsingular closed leaf. Once again, Figure 7 gives a picture which is far superior to the description of the sphere offered below.

Let us first choose a disk $D^{\prime} \subset \mathbb{S}^{3}$ such that

(1) $D_{\xi}^{\prime}$ is generic (section 3 );

(2) $\partial D^{\prime}=\Gamma$;

(3) $\left(V \cap D^{\prime}\right) \pitchfork \xi$ (the characteristic foliation is non-singular near the boundary);

(4) $\partial U \pitchfork D^{\prime}$;

(5) $\left(\partial U \cap D^{\prime}\right) \pitchfork \xi$

(6) $\partial V \pitchfork D^{\prime}$;

(7) $\left(\partial V \cap D^{\prime}\right) \pitchfork \xi$.

We can further assume that $D_{\xi}^{\prime}$ has only positive elliptic and negative hyperbolic singularities (see Lemma 3.8). In this case the absolute value of the self-linking number of $\Gamma$ is equal to the number of elliptic points plus the number of hyperbolic points. At the same time the number of hyperbolic points on the disk has to be exactly one less than the number of elliptic points. This means that $D_{\xi}^{\prime}$ must have both elliptic and hyperbolic singular points $(l(\Gamma)<-1)$.

Let $D=D^{\prime} \cap T$. By construction, all the singularities of $D_{\xi}^{\prime}$ are inside $T$; thus there are still hyperbolic and elliptic singularities on $D_{\xi}$. To make the description of the sphere easier, we can choose coordinates on $T, \widetilde{T}$ with the following properties:

(1) $T=\widetilde{T}=\{|z| \leq 1,|w|=1\} \subset\{z, w\}=\mathbb{C}^{2}$; 


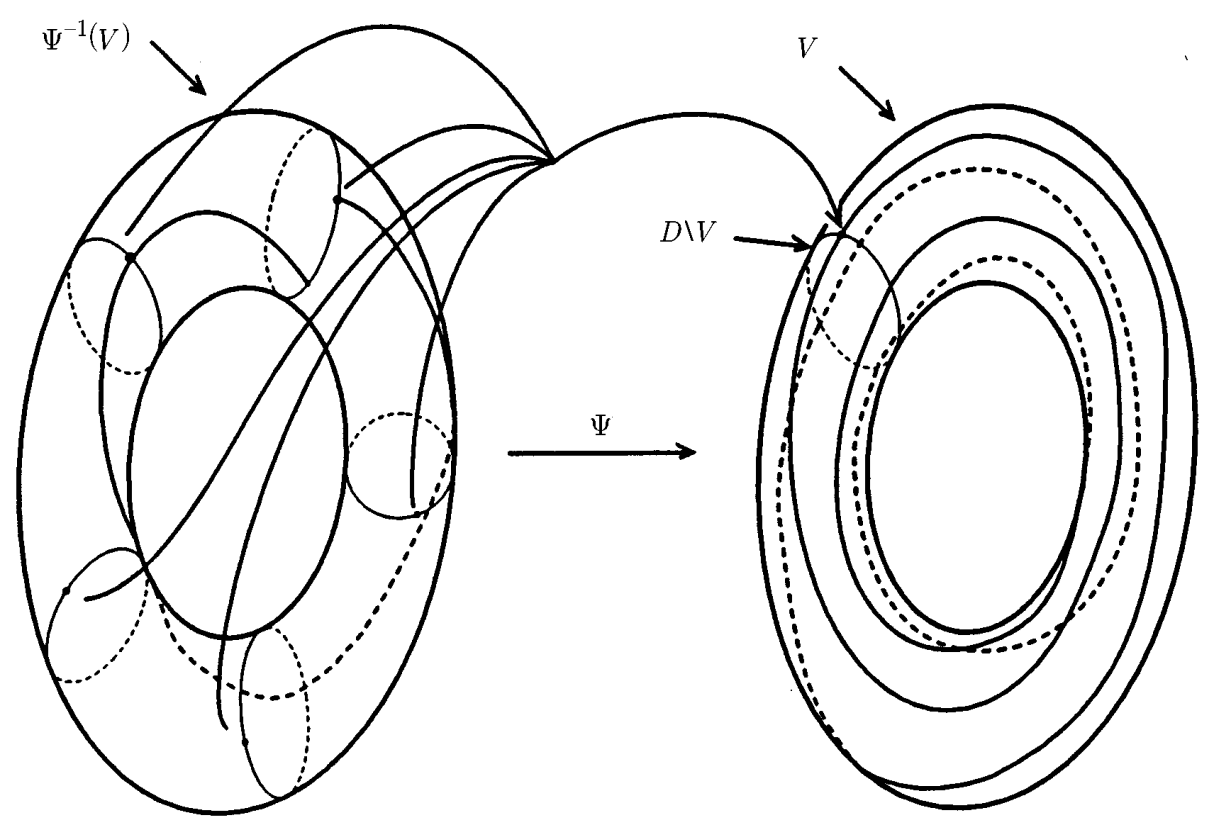

FIgURE 6 . The characteristic foliation of $\partial V$

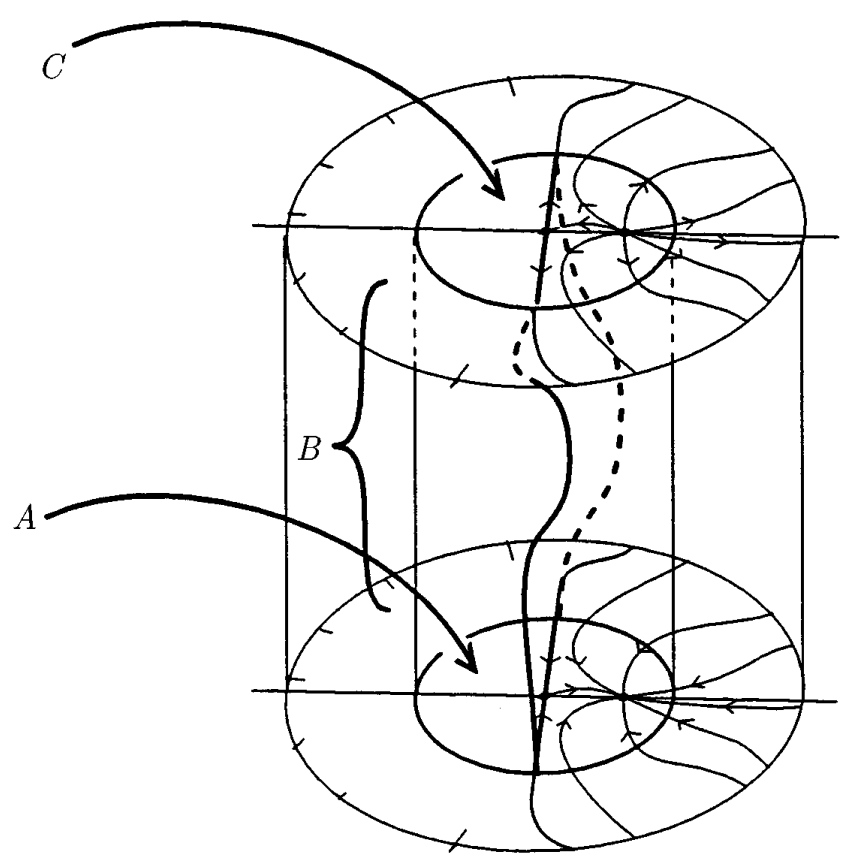

Figure 7. $A \cup B \cup C$ 
(2) $\psi(z, w) \mapsto\left(z, w^{q+1}\right)$;

(3) $D=\{w=1\} \subset T$;

(4) $\partial V=\{|z|=1 / 2\} \subset T$;

(5) the point $\{z=0, w=1\}$ is a (negative) hyperbolic singularity of $D_{\xi}$;

(6) the point $\{z=1 / 4, w=1\}$ is a (positive) elliptic singularity of $D_{\xi}$;

(7) the segment $\{i \cdot z \in[-3 / 4,3 / 4], w=1\}$ is a (singular) leaf of $D_{\xi}$.

Let

$$
\begin{aligned}
& A=\{|z| \leq 1 / 2, w=1\} \subset \widetilde{T}, \\
& B=\left\{|z|=1 / 2, w=e^{2 \pi i \theta}: 0 \leq \theta \leq \frac{q}{q+1}\right\} \subset \widetilde{T}, \\
& C=\left\{|z| \leq 1 / 2, w=e^{2 \pi \frac{q}{q+1}}\right\} \subset \widetilde{T} .
\end{aligned}
$$

Now consider

$$
F=A \cup B \cup C
$$

$F$ is a piecewise smooth sphere with transversal corners.

Let us now examine the characteristic foliation of $F: A$ and $C$ both cover the disk $D$; therefore their characteristic foliation is exactly like that of $D_{\xi}$. It is important to note, however, that if we assume that $A$ has the same orientation as $D$ and extend it to $F$, then $C$ will have the orientation opposite to that of $D$ which will reverse the orientation of the foliation as well. Now $B$ covers $\partial V$ exactly $q$ times. Therefore, the characteristic foliation of $B$ consists of Legendrian curves which start at some point $\left\{(1 / 2) \cdot e^{2 \pi i \theta}, 1\right\}$ and end at a point $\left\{(1 / 2) \cdot e^{2 \pi i \theta}, e^{2 \pi i \frac{q}{q+1}}\right\}$; in other words the characteristic foliation of $B$ connects points with the same $z$ coordinates. We now consider the negative hyperbolic point $\{z=0, w=1\}$ on $A_{\psi^{*}(\xi)}$. The two outgoing separatrices will have to exit through the boundary of $A$ (since $D$ was chosen not to have any negative elliptic points), so by the choice of the coordinates (see property (7)), these must exit through the points $\{z= \pm i \cdot 1 / 2, w=1\}$. The characteristic foliation of $B$ will connect these points at which these separatrices exit to the points $\left\{z= \pm i \cdot 1 / 2, w=e^{2 \pi i \frac{q}{q+1}}\right\}$ on the boundary of $C$ which (due to the reversal of orientation we mentioned) will be the entrance points of the incoming separatrices. These separatrices will lead us to the hyperbolic point $\left\{z=0, w=e^{2 \pi i \frac{q}{q+1}}\right\}$ on $C_{\psi^{*}(\xi)}$. This describes a closed leaf of $F_{\psi^{*}(\xi)}$ which passes through exactly two hyperbolic singularities. Now, a hyperbolic-hyperbolic connection is not generic; therefore, we can perturb the surface $F$ slightly to get a surface $F^{\prime}$ in which the outgoing separatrices of $\{z=0, w=0\}$ will go to the elliptic point $\left\{z=1 / 4, w=e^{2 \pi i \frac{q}{q+1}}\right\}$ on $C_{\psi^{*}(\xi)}$. But all the elliptic singularities of the characteristic foliation of $C$ are negative (due to the reversal of orientation). Thus we can smooth out $F^{\prime}$ using Lemma 2.9 and then we can apply the elimination lemma (see section 3 ) to produce the required sphere $S$.

Aside from providing an example of unusual behavior of contact structures, this example also has another consequence.

Corollary 9.2. If a Reeb vector field on the (tight) sphere has a contractible periodic orbit $\Gamma$ which spans a disk and is such that $1(\Gamma)<-1$, then there must exist another periodic orbit which is contractible in the complement of $\Gamma$. 
Proof. Hofer $[\mathrm{H}]$ has shown that on a closed manifold with an overtwisted contact structure, the Reeb vector field always has contractible periodic orbits. His results hold when applied to a manifold with boundary if the symplectization of that manifold has a pseudo-convex boundary (with respect to an almost-complex structure compatible with the symplectic form). It is also unnecessary to assume that the contact structure is overtwisted if it is known that some cover of the manifold has an overtwisted contact structure. These facts together with Theorem 9.1 imply the existence of another periodic orbit which has to be contractible in the complement of the given periodic orbit.

Section II. A tight contact structure which cannot be embedded into $\mathbb{S}^{3}$.

Theorem 9.3. There exists a tight contact structure $\hat{\xi}$ on $\mathbb{K}=\mathbb{R}^{2} \times \mathbb{S}^{1}$ which is not contactomorphic to any subset of $\left(\mathbb{S}^{3}, \xi\right)$ where $\xi$ is tight.

Proof. Once again, we shall first construct the necessary contact structure in Part I, and then show that it satisfies the required properties in Part II.

Part I - Construction: Consider a transversal curve $\Gamma \subset\left(\mathbb{S}^{3}, \xi\right)$ such that $l(\Gamma)=$ -1 . As we mentioned in the previous theorem, there exists a tubular neighborhood of $\Gamma$ which is contactomorphic to the torus $\left\{r<(2 \pi)^{-2}\right\} \in \mathbb{T}$. Let $K$ be that neighborhood.

Consider now another transversal curve $\Gamma^{\prime} \subset K$ such that $l\left(\Gamma^{\prime}\right)=-3$ and $\Gamma^{\prime}$ is isotopic to $\Gamma$ inside $K$ (such curves are known to always exist). This curve also has a tubular neighborhood contactomorphic to $\left\{r<(2 \pi)^{-2}\right\} \in \mathbb{T}$; this neighborhood can be chosen to lie inside $K$. Call that neighborhood $K^{\prime}$.

Note that $\bar{K}$ is contactomorphic to $\overline{K^{\prime}}$. Let $M=\bar{K} \backslash K^{\prime}$. Let $K_{0}=K^{\prime} \cup M=$ $\bar{K} \cong \overline{K^{\prime}}$ and define $K_{n}=K_{n-1} \cup M . \mathbb{K}=\bigcup_{n} K_{n}$ is the necessary contact manifold.

Part II: It is clear that the contact structure thus constructed is tight since $K_{n}$ is contactomorphic to $\bar{K}$ for every $n$ and $K$ is tight.

We now proceed with showing that the manifold we obtained cannot be imbedded into $\mathbb{S}^{3}$.

Note that any transversal curve on the boundary of $K$ must be transversally isotopic to $\Gamma$ and any transversal curve on the boundary of $K^{\prime}$ must be transversally isotopic to $\Gamma^{\prime}$. This means that if we could calculate the self-linking number of curves isotopic to $\Gamma$ we would see that any transversal curve on the "outer boundary component" of $M$ would have a self-linking number higher by two than the selflinking number of a transversal curve on the "inner boundary component". In particular if there was an imbedding of $\phi: \mathbb{K} \rightarrow \mathbb{S}^{3}$, then it would be possible to find a curve isotopic to $\phi(\Gamma)$ with an arbitrarily high self-linking number, but that contradicts the Bennequin inequality. This means that $K_{\infty}$ cannot be embedded into $\mathbb{S}^{3}$.

\section{ACKNOWLEDGEMENTS}

First, I would like to thank my advisor, Professor Yakov Eliashberg, for his patience and support and for his guidance.

In addition, I would like to acknowledge the support I received from Institute Henri Poincaré where part of this research was carried out. I would also like to thank Professor Claude Viterbo for his hospitality. 
Finally I would especially like to thank my extended family; without them I would not be where I am today.

\section{REFERENCES}

[A1] V. I. Arnold, Mathematical Methods of Classical Mechanics, MIR, Moscow, 1974.

[A2] V. I. Arnold, Geometrical Methods in the Theory of Ordinary Differential Equations, Nauka, Moscow, 1978.

[AG] V. I. Arnold, A. Givental, Symplectic Geometry, Dynamic Systems IV.

[AMR] R. Abraham, J. E. Marsden and T. Raitu, Manifolds, Tensor Analysis, and Applications, Springer-Verlag, New York, 1988. MR 89f:58001

[Be] D. Bennequin, Entrelacement et equations de Pfaff, Asterisque 107-108 (1983), 87-161. MR 86e: 58070

[El1] Ya. Eliashberg, Contact 3-Manifolds Twenty Years Since J. Martinet's Work, Ann. Inst Fourier (Grenoble) 42 (1992), 1-12. MR 93k:57029

[El2] Ya. Eliashberg, New Invariants of Open Symplectic and Contact Manifolds, J. Amer. Math. Soc. 4 (1991), 513-520. MR 92c:58030

[El3] Ya. Eliashberg, Classification of overtwisted contact structures on 3-manifolds, Invent. Math. 98 (1989), 623-637. MR 90k:53064

[El4] Ya. Eliashberg, Legendrian and transversal knots in tight contact 3-manifolds, Topological Methods in Modern Mathematics (1993), 171-193. MR 94e:57005

[Gi1] E. Giroux, Convexite en Topologie Contact, Comment. Math. Helv. 66 (1991), 637-677. MR 93b:57029

[Gi2] E. Giroux, Une structure de contacte, meme tendue, est plus ou moins tordue, Ann. Ecole Norm. Sup. 27 (1994), 697-705. MR 96b:57034

[Gi3] E. Giroux, Classification of tight contact structures on a three-torus, to appear.

[Gr] J. W. Gray, Some global properties of contact structures, Ann. of Math. 69 (1959), 421450. MR 22:3016

$[\mathrm{H}] \quad$ H. Hofer, Pseudoholomorphic curves in symplectizations with applications to the Weinstein conjecture in dimension three, Invent. Math. 114 (1993), 515-564. MR 94j:58064

[KO] Y. Katznelson and D. Ornstein, The differentiability of the conjugation of certain diffeomorphisms of the circle, Ergod. Th. \& Dynam. Sys. 9 (1989), 643-680. MR 91i:58121

[Mo] J. Moser, On the volume elements on a manifold, Trans. Amer. Math. Soc. 120 (1969), 286-294. MR 32:409

[Si] C. L. Siegel, Note on differential equations on the torus, Ann. of Math. 46 (1945), 423-428. MR 7:117g

Département de Mathématiques, Université du Québec À Montréal, Montréal, CANADA

E-mail address: sergei@math.uqam.ca 